\title{
Innovación y productividad en las empresas manufactureras ecuatorianas*
}

\author{
Jorge Antonio Rodríguez Moreno \\ Universidad Santa María, Ecuador \\ María Engracia Rochina Barrachina \\ Universitat de València y ERICES
}

\section{Resumen}

En este trabajo se estudia la relación entre la I+D de las empresas y su productividad utilizando datos del Censo Económico de Ecuador, 2010, y un modelo à la Crépon-Duguet-Mairesse (CDM, 1998). Es decir, se incluye un probit para la decisión de I+D, un modelo de Heckman para la inversión en $I+D$, y un regresor de gasto en $I+D$ corregido por endogeneidad en las regresiones de productividad. Se utilizan distintas medidas de productividad (del trabajo y Productividad Total de los Factores). Los resultados son robustos a las distintas medidas de productividad, e indican que las empresas manufactureras ecuatorianas que invierten en $I+D$ presentan mayores niveles de productividad.

Palabras clave: empresas manufactureras ecuatorianas, $I+D$, productividad.

Clasificación JEL: L6, O54, O3, D24.

\begin{abstract}
This paper studies the relationship between firms' $R \& D$ activities and their effects on productivity. The empirical analysis uses data from the Ecuador Economic Census, 2010. The paper is done in the spirit of the Crépon-Duguet-Mairesse (CDM, 1998) model. That is, it includes a probit for the $R \& D$ decision, a Heckman's model for $R \& D$ expenditures and an endogeneity corrected $R \& D$ expenditure regressor in the productivity regressions. We use different measures of firms' productivity either based on simple ratios of labour productivity or on Total Factor Productivity estimates. Our results are robust to different productivity measures, and indicate that those Ecuadorian firms investing in $R \& D$ exhibit higher productivity levels.
\end{abstract}

Keywords: Ecuadorian manufacturing firms, $R \& D$, productivity

JEL classification: L6, O54, O3, D24.

* Los autores agradecen los comentarios y sugerencias recibidos del editor de este número de la revista, Gustavo Nombela Merchán, y de un evaluador anónimo. Este trabajo ha sido financiado por el Ministerio español de Economía y Competitividad por medio del proyecto de investigación ECO2014-55745-R; Fundación BBVA, dentro del Programa de Ayudas a Proyectos de Investigación en Socioeconomía, 2014; la Generalitat Valenciana (programa PROMETEOII/2014/054); la Secretaría de Educación Superior, Ciencia, Tecnología e Innovación de Ecuador por medio de su programa de Becas Convocatoria abierta 2011; y la Universidad Santa María, Campus Guayaquil, por su aporte mediante los fondos de investigación periodo 2014-2019. 


\section{Introducción}

En los últimos años se ha escrito mucho sobre el crecimiento económico y los factores que lo originan o lo causan. Solow (1957) planteaba que uno de los factores fundamentales para el crecimiento económico estaba relacionado con los cambios tecnológicos realizados en los países por parte de las empresas, y que eran conducentes a aumentos en productividad. Posteriormente, Aghion y Howitt $(1992 ; 1998)$ desarrollarían modelos que confirman dichas ideas y que introducen la Investigación y el Desarrollo (I+D) como parte de estos cambios y como complemento de las ideas preliminares de Schumpeter (1942) sobre la «destrucción creativa». Entre los trabajos empíricos que analizan la relación entre la I+D y la productividad se encuentran los de Griliches (1970; 1979), Doraszelski y Jaumandreu (2013), Máñez et al. (2006), y Añón y Manjón (2009). En ellos se encuentra evidencia empírica de una relación positiva entre la productividad de las empresas y su realización de actividades de I+D. Algunos argumentos relevantes que justifican esta relación son los siguientes (Hall, 2011): por un lado, las empresas que invierten en I+D pueden tanto aumentar su eficiencia productiva como mejorar los productos que ofrecen, aumentando su demanda así como reduciendo sus costes de producción. Por otro lado, las empresas que hacen $\mathrm{I}+\mathrm{D}$ presentan previsiblemente mejores perspectivas de crecimiento, lo que puede conducir a un mejor aprovechamiento de economías de escala en la producción, con la consiguiente reducción en costes.

Dentro de la línea de análisis que se ocupa de la relación entre la productividad, la innovación y la inversión en $\mathrm{I}+\mathrm{D}$, se encuentra el trabajo seminal de Crépon et al. (1998) que, utilizando información de corte transversal de las empresas manufactureras en Francia, desarrolla un modelo llamado CDM (por la inicial de los apellidos de sus autores) que explica las relaciones implicadas utilizando varias etapas de estimación: una primera etapa en la que se estima la inversión en I+D, una segunda etapa en la que se estiman los resultados innovadores de la inversión en I+D y, por último, la estimación de la productividad de las empresas en función de sus actividades innovadoras. Siguiendo esta línea de trabajo, Crespi y Zúñiga (2012) realizan un análisis conjunto para Argentina, Chile, Colombia, Costa Rica, Panamá y Uruguay, en el que encuentran que implementar nuevas tecnologías mejora la productividad. Sin embargo, Benavente (2006), en un estudio para Chile, no encuentra un impacto en la productividad derivado de la innovación. Así mismo, Hall (2011) realiza una revisión de artículos donde se emplea la metodología CDM en la que, además de encontrarse trabajos para los países antes citados, también se revisan trabajos para Alemania, España, Reino Unido, Italia, Suecia, China, Finlandia, Noruega, Estonia y Holanda. De esta revisión se deduce que no siempre se encuentra empíricamente una relación entre productividad e innovación de las empresas. En particular, de la revisión de Hall (2011) parece desprenderse que el resultado es más claro para trabajos que distinguen entre innovación de producto y de proceso, con la peculiaridad de que se encuentran más casos de significatividad 
estadística en la relación entre innovación de producto y productividad que en lo referente a la innovación de proceso.

El objetivo de este trabajo es arrojar más luz sobre la relación entre la actividad innovadora de las empresas y su productividad a través del análisis de un país en desarrollo como es Ecuador. Para ello, se utilizará el Censo Económico de Ecuador 2010 en lo referente al sector manufacturero. En dicho censo consta información sobre la inversión en I+D de las empresas y sobre otras características generales de las mismas. Si bien no existe información sobre los resultados de los procesos de inversión en I+D en términos de innovaciones de producto, proceso y/o patentes, se puede considerar a la inversión en I+D y a su cuantía como una proxy razonable de la actividad innovadora desarrollada por las empresas. La escasa utilización de la citada base de datos para el análisis empírico no meramente descriptivo convierte este trabajo en novedoso y pionero para Ecuador. En primer lugar, se busca explicar qué determina la decisión de I+D de las empresas manufactureras en Ecuador, lo que se hará mediante un modelo probit para variable dicotómica que explique la probabilidad de tal decisión. En segundo lugar, se está interesado en estimar una ecuación para explicar los gastos de I+D de las empresas, lo que se hará mediante la estimación de un modelo de selección de muestra. Por último, y en tercer lugar, se pretende averiguar si hay evidencia para dicho país sobre la existencia de una relación significativa y positiva entre la realización de actividades de I+D y la productividad de las empresas, medida esta a través de distintos indicadores de productividad que se pueden obtener con datos de corte transversal, como son las ventas y el Valor Añadido por trabajador, así como la Productividad Total de los Factores (PTF), tanto en su versión de producción como de Valor Añadido.

El principal resultado en este trabajo es la constatación de una relación positiva y significativa de los gastos de I+D de las empresas sobre su productividad y, al mismo tiempo, que una serie de actividades también realizadas por algunas empresas y que están ligadas a prácticas empresariales más profesionales y modernas contribuyen de forma directa y/o indirecta (a través del gasto en I+D) a las mejoras de productividad de las empresas ecuatorianas.

El trabajo está estructurado del siguiente modo. La sección 2 presenta la base de datos utilizada y realiza un análisis descriptivo de la misma en relación a ciertas variables de interés en el estudio. La sección 3 explica las distintas etapas de estimación y los resultados asociados a cada una de ellas. Así, la sección 3.1 contiene la explicación del modelo probit ejecutado con la variable dicotómica de inversión o no en I+D, la sección 3.2 se ocupa del modelo de selección de muestra de Heckman (1979) para estimar correctamente y sin sesgo la ecuación del gasto en I+D de las empresas y su predicción y, por último, la sección 3.3 contiene la ecuación de regresión que liga la productividad de las empresas al ejercicio de su actividad de I+D. La sección 4 recoge las principales conclusiones del trabajo. 


\section{Datos y análisis descriptivo}

Para la elaboración de este trabajo se ha utilizado el Censo Económico de Ecuador 2010. Esta es una encuesta que fue realizada a 511.130 empresas y donde la mayoría de preguntas hacen referencia a información del año 2009. Dentro de esta base se encuentra información acerca de distintas características de las empresas como, por ejemplo, su edad, localización, situación jurídica, sectores a los que pertenecen, número de trabajadores, ventas y principales clientes, costes, ingresos y activos fijos, que forman, entre otras, parte del grupo de variables con el que se va a trabajar.

En este trabajo, centrado en manufacturas, se utilizarán solo las empresas manufactureras, lo que determina un número de 44.109 empresas ${ }^{1}$. Tras la eliminación de empresas con falta de información para variables relevantes en nuestro análisis, este número de empresas se reduce a 42.292. La información sobre pertenencia sectorial de las mismas se encontraba altamente desagregada, por lo que se realizó una agrupación mayor que lleva a trabajar finalmente con 12 sectores (véase Anexo 1$)^{2}$.

En nuestro análisis descriptivo el primer paso consiste en describir las decisiones de realización de actividades de I+D por parte de las empresas. Existe para ello una pregunta específica en la base de datos utilizada que permite la construcción de una variable dummy que toma valor 1 para las empresas que invierten en $\mathrm{I}+\mathrm{D}$ y 0 en caso contrario. A partir de esta pregunta se obtiene que las empresas que realizan I+D son 412, lo que representa tan solo el 0,97 por 100 de las empresas manufactureras. Por tanto, dentro del sector manufacturero en Ecuador, se trata de una actividad muy poco extendida.

En segundo lugar, también se realiza un análisis descriptivo comparativo entre las empresas que invierten en I+D y aquellas que no lo hacen. Este análisis se basa en una serie de indicadores relacionados con la productividad como son las ventas por trabajador, la intensidad de capital por trabajador, el uso de materiales por trabajador y el tamaño de la empresa. Estos indicadores han sido transformados a una escala logarítmica. A partir de ellos se ejecuta un contraste de medias entre sus valores para empresas que invierten y que no invierten en I+D. Los resultados del proceso de contraste se muestran en el Cuadro 1. El contraste calcula, para cada indicador, si la diferencia de medias asociada a cada una de las dos decisiones consideradas es estadísticamente distinta de 0 . Por ejemplo, se calculan las ventas por trabajador para todas las empresas y se compara la media de esta variable para las empresas

\footnotetext{
${ }^{1}$ Además del sector manufacturero, el Censo Económico de Ecuador 2010 incluye la información de otros sectores como: Servicios, que representa el 87,12 por 100 de las empresas (Comercio al por mayor y al por menor representa el 59,9 por 100 del total de servicios); y Agricultura, Extracción de Recursos Naturales, Construcción y Suministros de Agua y de Gas, que agrupan menos del 1 por 100 de las empresas cada uno de ellos.

${ }^{2}$ Ante la falta de trabajos empíricos con esta base de datos, el agrupamiento se realizó siguiendo como ejemplo otros agrupamientos realizados en otros trabajos similares para otros países centrados en el estudio de manufacturas (véase, por ejemplo, MÁÑEZ et al., 2015).
} 


\section{CUADRO 1}

CONTRASTES DE DIFERENCIA DE MEDIAS ENTRE EMPRESAS QUE INVIERTEN VERSUS EMPRESAS QUE NO INVIERTEN EN I+D

\begin{tabular}{|c|c|c|c|c|}
\hline & $\mathbf{I}+\mathbf{D}$ & No I+D & $\begin{array}{l}\text { Diferencia de } \\
\text { medias }^{1}\end{array}$ & $P$-value ${ }^{2}$ \\
\hline Ventas por trabajador & 10,132 & 8,834 & $1,299 * * *$ & 0,000 \\
\hline Capital por trabajador & 8,885 & 7,343 & $1,542 * * *$ & 0,000 \\
\hline Materiales por trabajador & 9,012 & 7,850 & $1,162 * * *$ & 0,000 \\
\hline Número de empleados & 85,616 & 4,548 & $81,068 * * *$ & 0,000 \\
\hline
\end{tabular}

NOTAS: ***Indica significatividad estadística al 1\%. 1. Calculada como diferencia Media Empresas I+D Media Empresas No-I+D. 2. $\mathrm{H}_{0}=\mathrm{La}$ diferencia de las medias es igual a 0.

que realizan I+D (valor 1 de la dummy correspondiente) con la media de las que no realizan dicha actividad (valor 0 de la dummy correspondiente). Si la diferencia es estadísticamente distinta de 0 , esto implica que hay diferencias en la variable de ventas por trabajador entre los dos tipos de empresas. Además, el resultado del contraste también nos proporciona el signo de la diferencia.

La implementación de este tipo de contrastes muestra que existe una relación entre los indicadores mencionados y las decisiones de I+D. Para todos los indicadores se obtiene que realizar actividades de I+D está asociado a valores medios superiores. Por lo tanto, las empresas que invierten en I+D tienen un mayor volumen de ventas, capital y materiales por trabajador, así como son de mayor tamaño (medido este por el número de trabajadores).

\section{Metodología de estimación y resultados}

La metodología de estimación utilizada en este trabajo comprende distintas etapas. La primera de ellas consiste en estimar la probabilidad de realización de actividades de I+D por parte de las empresas. Esto se realizará mediante un modelo probit univariante. La segunda consiste en utilizar el modelo de selección de muestra de Heckman (1979) con el fin de estimar sin sesgo una ecuación de gasto en I+D de las empresas y obtener la predicción del mismo. Por último, en la tercera etapa se realiza el análisis de regresión que conduce a la estimación de la relación existente entre la productividad de las empresas y su implicación en actividades de I+D.

\subsection{Metodología de estimación y resultados del Probit univariante}

Los modelos de elección discreta aparecen cuando la variable dependiente objeto de estudio toma valores que tienen esa característica. Dentro de este grupo de 
modelos se encuentran los modelos binarios, que indican que la variable dependiente puede tomar solo dos valores. Por ejemplo, 1 ó 0 en el caso de ser afirmativa o negativa la ocurrencia del evento reflejado en la variable dependiente. En nuestro caso particular, se pretende obtener el efecto de las variables explicativas (vector $X_{i}$ ) sobre la probabilidad de que las empresas inviertan o no en I+D $\left(I+D_{i}\right)$. Si los errores subyacentes al modelo de decisión $\left(\varepsilon_{i}\right)$ siguen una distribución normal $(F)$, se tiene el modelo Probit:

$$
\begin{aligned}
& I+D_{i}= \begin{cases}1 & \text { Si } \beta_{0}+x_{i}^{\prime} \beta_{1}+\varepsilon_{i} \\
0 & \text { Caso contrario }\end{cases} \\
& \operatorname{Prob}\left(I+D_{i}=1\right)=F\left(\beta_{0}+x_{i}^{\prime} \beta_{1}\right) \\
& F\left(\beta_{0}+x_{i}^{\prime} \beta_{1}\right)=\int_{-\infty}^{\beta_{0}+x_{i}^{\prime} \beta_{1}} \frac{1}{\sqrt{2 \pi}} e^{-t^{2} / 2} d t
\end{aligned}
$$

Las variables explicativas incluidas en el modelo para explicar la decisión de inversión en I+D se definen en el Anexo 2. Primero, se han considerado tres grupos de variables explicativas que caracterizan estructuralmente a las empresas desde el punto de vista de su ubicación geográfica, el sector manufacturero al que pertenecen y su forma jurídica. La ubicación geográfica de la empresa se introduce a través de variables dummy para cada provincia del país. Con ello se pretende controlar por características inherentes a las provincias que determinen una mayor o menor propensión a invertir en $\mathrm{I}+\mathrm{D}$ por parte de las empresas (una vez que las características de las empresas han sido controladas en la estimación).

En el país existen 24 provincias, de las cuales tres de ellas (Pichincha, Guayas y Azuay) concentran el 53,7 por 100 de las empresas manufactureras, lo que podría hacer pensar que tal vez son las provincias más propensas a realizar $\mathrm{I}^{+} \mathrm{D}^{3}$. Por lo que respecta al sector manufacturero al que pertenecen las empresas (véase Anexo 1), este ha sido controlado en la estimación por medio de la inclusión de variables dummy para cada sector. Con ello se pretende identificar si hay efectos sectoriales más allá de las características de las empresas que resultan significativos a la hora de explicar la probabilidad de que una empresa invierta o no en I+D. Las distintas formas jurídicas que en la base de datos aplican al sector manufacturero son 9 (véase Anexo 2). Cabe aclarar en este punto que hay ciertos tipos de empresas atendiendo a su forma jurídica que podrían interpretarse como de servicios. Sin embargo, dado que se trata de empresas que pertenecen a manufacturas por la clasificación sectorial, puede que determinadas empresas, por diferentes razones, teniendo un tipo de clasificación jurídica cuyo nombre las acerca a una actividad de servicios,

\footnotetext{
${ }^{3}$ Existe, además, una categoría de «provincia» denominada zona no delimitada para territorios en conflicto.
} 
realicen actividades manufactureras ${ }^{4}$. Los tipos de empresas que a priori podrían considerarse más proclives a la inversión en I+D son tanto las empresas clasificadas como privadas y las clasificadas como de control extranjero. Las privadas, debido a su fin de maximización de beneficios, ya sea por medio de un aumento de su producción o por la minimización de costes, y las de control extranjero, debido tanto a que la experiencia en el país de origen puede ser transmitida a la filial o a que, según el trabajo de Añón et al. (2011), las multinacionales presentan mayores retornos sobre la inversión en $\mathrm{I}+\mathrm{D}$.

Además de las variables explicativas que se ha considerado que caracterizan estructuralmente a las empresas, se han tomado en consideración un grupo de variables con las que se pretende captar el mayor o menor grado de profesionalización/ modernización de la empresa en lo referente a la calidad y diligencia de la misma en su gestión y prácticas empresariales. Las variables consideradas son, en primer lugar, si la empresa pertenece o no a una red o grupo empresarial, cuyo efecto sobre la innovación a nivel empírico no siempre es coincidente (véanse los trabajos de Chudnovsky et al., 2006, y Parisi et al., 2006, que no encuentran un efecto significativo sobre la innovación de producto o proceso, mientras que Hall y Mairesse, 2009, sí que lo encuentra sobre la intensidad de gasto en I+D). En segundo lugar, si la empresa realiza trabajos de investigación de mercado. En tercer lugar, si la empresa lleva a cabo contabilidad; en cuarto lugar, si la empresa tiene acceso al financiamiento externo; en quinto lugar, si la empresa realiza actividades de mejora medioambientales.

En relación con esta última variable, Porter y Van der Linde (1995) planteaban que las mejoras ambientales generadas por las empresas ocasionan en muchas ocasiones productos de mejor calidad, o de menor coste (por el uso de materias primas más baratas), lo que se traduce en una mayor competitividad de las empresas y es al mismo tiempo beneficioso para la innovación. Sin embargo, Ambec et al. (2013) revisan esta cuestión de forma más exhaustiva en la literatura y concluyen que esta asociación entre el ejercicio de actividades medioambientales y la innovación no siempre existe.

Por otro lado, se tiene en cuenta en sexto lugar si la empresa llevó a cabo algún programa de capacitación o formación para sus empleados. Thornhill (2006) mostró que este tipo de actividades son beneficiosas para la innovación de las empresas manufactureras dependiendo del dinamismo del sector donde se ubiquen. En séptimo lugar, si la empresa hace uso de las tecnologías de la información y de la comunicación (TIC). Ollo-López y Aramendía-Muneta (2012) encuentran ciertos efectos de las TIC sobre la innovación dependiendo del nivel de desagregación con que se cuente en los datos sobre el tipo de TIC implementada. Por último, si el principal cliente

${ }^{4}$ Puede darse el caso de una empresa que se dedique a actividades de manufactura sin estar autorizada por su razón social o forma jurídica. Pero en ese caso, la oficina de Estadística debe tomarla en consideración al realizar la encuesta, ya que debe reflejar la actividad realizada y no solo la registrada oficialmente en los entes gubernamentales correspondientes. Por otro lado, existe el caso de las Cooperativas de Ahorro y Crédito (algo similar a las Cajas de Ahorro que existen en algunos países), que por impago de un crédito por parte de una empresa manufacturera pueden hacer efectivas las garantías que se entregaron para el mismo. Esto puede conllevar a que pasen a ser propietarias de bienes productivos como fábricas, plantas industriales y demás activos, entre otros, que pasarían a ser administrados por esta institución. 
de la empresa es extranjero, en cuyo caso la empresa exporta. A pesar de ser un indicador incompleto sobre la actividad exportadora de las empresas manufactureras ecuatorianas, en la medida en que las empresas que contestan positivamente a esta pregunta son por definición exportadoras, la apertura a los mercados internacionales y su grado de competencia puede incentivar la actividad innovadora de las empresas. Así, por ejemplo, Bratti y Felice (2012) muestran que existe un efecto positivo de la actividad exportadora de las empresas sobre su actividad innovadora tanto de producto como de proceso.

Adicionalmente, existen otras variables explicativas de control, que han sido consideradas e incorporadas de forma dicotómica, como son: la calificación artesanal ${ }^{5}$, si el local es propio, si la empresa encuestada coincide con la matriz y si el gerente de la empresa es hombre. Por último, existen dos controles adicionales: por una parte, la edad de la empresa (en logaritmos) se utiliza como proxy de su experiencia, pero también puede captar cuestiones relacionadas con el ciclo de vida del producto y, por otra parte, el número de trabajadores (en logaritmos) y su cuadrado correspondiente, utilizados como medida del tamaño de las empresas. Siguiendo la línea seminal en el trabajo de Schumpeter (1942), se espera que las empresas más grandes sean las más propensas a innovar ${ }^{6}$.

Finalizada la enumeración de las variables explicativas, su efecto sobre la decisión de inversión en I+D de las empresas se estima por medio de la estimación de un modelo probit aplicado a la expresión [1]. Dicho modelo especifica la probabilidad de invertir frente a no invertir en I+D dados los valores de las explicativas. Su estimación presenta tanto un interés per se como el interés de formar parte de la siguiente etapa de estimación en este trabajo, donde se estimará una función de gasto en I+D de las empresas tomando en consideración el posible problema potencial de sesgo por selección de muestra.

Para obtener los coeficientes $(\beta)$ estimados, en estos modelos se utiliza una función de verosimilitud sobre la decisión de invertir en I+D. Dicha función viene típicamente expresada en logaritmos:

$$
\log L=\sum_{\left(I+D_{i}\right)}\left[\left(I+D_{i}\right) \log \left[F\left(x_{i}^{\prime} \beta\right)\right]+\left(1-\left(I+D_{i}\right)\right) \log \left[1-F\left(x_{i}^{\prime} \beta\right)\right]\right]
$$

donde $\left(I+D_{i}\right)$ es la variable dicotómica que toma valor 1 si la empresa invierte en $\mathrm{I}+\mathrm{D}$ y valor 0 en caso contrario.

Una vez obtenida la función de verosimilitud, esta se maximiza con respecto a $\beta$ :

$$
\operatorname{Max}_{\beta} \log L \rightarrow \frac{\partial \log L}{\partial \beta}=0
$$

${ }^{5}$ La Ley de Defensa del Artesano (1998) establece algunos parámetros para que las personas que estén dedicadas a esta actividad (artesanal), sean partícipes de ciertos beneficios tributarios. Se trata en esencia de personas naturales (similar a los autónomos que existen en varios países) que pueden obtener esta calificación y que se corresponden con pequeñas empresas por su inversión en bienes de capital y número de empleados.

${ }^{6}$ La información descriptiva de todas las variables incluidas en este trabajo se encuentra en el Anexo 3. 
Los resultados de la estimación de los efectos marginales medios correspondientes al modelo probit se presentan en el Cuadro 2. Sobre las variables de ubicación geográfica (para las que la categoría de referencia es Pichincha) se obtiene que pertenecer a la provincia de Imbabura tiene un efecto positivo y significativo sobre la decisión de invertir en I+D. Por el contrario, provincias como El Oro, Guayas y Loja presentan coeficientes negativos y significativos ${ }^{7}$. En relación a los sectores manufactureros, donde la categoría de referencia es el sector de AlimentaciónBebidas-Tabaco, los sectores que presentan una mayor probabilidad de invertir en I+D son el Químico, el de Maquinaria de Oficina-Electrónica y Otra Maquinaria Especializada, y el de Equipos de Comunicación-Precisión y Equipos Médicos, todos ellos sectores clasificados por la OCDE como de media y alta tecnología y, por tanto, más intensivos en I+D. Sin embargo, sectores como el Textil, el de la Madera y Papel, el de Productos no Metálicos, el de Productos Metálicos, y el del Mueble, presentan un efecto negativo sobre la decisión de invertir en I+D. Por lo que respecta a la forma jurídica de las empresas, ninguna forma jurídica resulta estadísticamente significativa en la decisión de $\mathrm{I}+\mathrm{D}$ (en relación a la categoría de referencia que es el grupo mayoritario de empresas adscritas a personas naturales) ${ }^{8}$.

En cuanto al grupo de variables con las que se pretende captar el grado de profesionalización/modernización de la empresa en el ejercicio de sus prácticas empresariales, los resultados del Cuadro 2 indican que todas las variables, a excepción de la que indica que el principal cliente de la empresa es extranjero, son tanto estadísticamente significativas como positivas. Para ilustrar la magnitud de los efectos marginales asociados a estas variables tómese, como ejemplo, el efecto marginal estimado $(0,0188)$ para la variable dummy de inversión en formación de la mano de obra de la empresa. Si la empresa pasara de no invertir a invertir en formación, su probabilidad media estimada de invertir en $\mathrm{I}+\mathrm{D}$, que es en términos unitarios de 0,009 , pasaría a ser de 0,0278 .

Por último, la variable de control que captura el tamaño de la empresa tiene un efecto también positivo y estadísticamente significativo. Este último resultado es favorable a las ideas de Schumpeter sobre la relación existente entre el tamaño de la empresa y la innovación.

\footnotetext{
${ }^{7}$ No se han podido estimar los coeficientes asociados a las provincias de Morona Santiago, Galápagos, Santa Elena y la categoría de zonas no delimitadas, debido a que en cada una de estas provincias la totalidad de las empresas en nuestros datos no presenta gastos positivos en I+D, lo que provoca en la estimación un problema de predicción perfecta de ceros. Las empresas ubicadas en estas zonas representan aproximadamente el 2,6 por 100 del total de manufacturas.

${ }^{8}$ No se han podido estimar los coeficientes asociados a las formas jurídicas Empresas sin Fines de Lucro, Empresas bajo Control Extranjero, Empresas de Gobierno Local, y Cooperativas de Ahorro y Crédito, debido a que para cada una de estas formas jurídicas la totalidad de las empresas no realiza actividades de I+D, lo que provoca en la estimación un problema de predicción perfecta de ceros. Las empresas bajo estas formas jurídicas representan aproximadamente el 0,14 por 100 del total de manufacturas.
} 
CUADRO 2

LAS DECISIONES DE INVERSIÓN Y DE GASTO EN I+D

\begin{tabular}{|c|c|c|c|c|}
\hline & \multirow[b]{2}{*}{ Variables } & \multirow{2}{*}{$\begin{array}{c}\text { Si/No I+D } \\
\text { y }=\text { Prob. pred } \\
(\mathrm{I}+\mathrm{D}=\mathbf{1})=\mathbf{0 , 0 0 9} \\
\text { dy/dx } \\
\text { (Efectos marg. medios) }\end{array}$} & \multicolumn{2}{|c|}{ Gasto en I+D } \\
\hline & & & Heckman & MCO \\
\hline \multirow[t]{16}{*}{$\begin{array}{l}\text { Ubicación geográfica de } \\
\text { la empresa }\end{array}$} & Azuay & $\begin{array}{c}0,000774 \\
(0,593)\end{array}$ & $\begin{array}{c}0,220 \\
(0,435)\end{array}$ & $\begin{array}{c}0,228 \\
(0,449)\end{array}$ \\
\hline & Bolívar & $\begin{array}{c}0,00538 \\
(0,363)\end{array}$ & $\begin{array}{c}0,460 \\
(0,213)\end{array}$ & $\begin{array}{c}0,519 \\
(0,184)\end{array}$ \\
\hline & Cañar & $\begin{array}{c}-0,00250 \\
(0,421)\end{array}$ & $\begin{array}{l}-0,889 \\
(0,458)\end{array}$ & $\begin{array}{l}-0,922 \\
(0,474)\end{array}$ \\
\hline & Carchi & $\begin{array}{c}0,00138 \\
(0,831)\end{array}$ & $\begin{array}{c}1,403 * * * \\
(0,003)\end{array}$ & $\begin{array}{c}1,415^{* * * *} \\
(0,004)\end{array}$ \\
\hline & Cotopaxi & $\begin{array}{c}-0,000627 \\
(0,819)\end{array}$ & $\begin{array}{l}-0,458 \\
(0,474)\end{array}$ & $\begin{array}{l}-0,461 \\
(0,501)\end{array}$ \\
\hline & Chimborazo & $\begin{array}{c}0,000450 \\
(0,855)\end{array}$ & $\begin{array}{l}-0,570 \\
(0,158)\end{array}$ & $\begin{array}{l}-0,568 \\
(0,187)\end{array}$ \\
\hline & El Oro & $\begin{array}{c}-0,00816^{* * *} \\
(0,000)\end{array}$ & $\begin{array}{c}-1,388 * * * \\
(0,005)\end{array}$ & $\begin{array}{c}-1,599 * * * \\
(0,001)\end{array}$ \\
\hline & Esmeraldas & $\begin{array}{c}0,000751 \\
(0,853)\end{array}$ & $\begin{array}{c}0,555 \\
(0,597)\end{array}$ & $\begin{array}{c}0,568 \\
(0,605)\end{array}$ \\
\hline & Guayas & $\begin{array}{c}-0,00259^{* *} \\
(0,017)\end{array}$ & $\begin{array}{c}0,048 \\
(0,849)\end{array}$ & $\begin{array}{c}0,011 \\
(0,966)\end{array}$ \\
\hline & Imbabura & $\begin{array}{c}0,00762 * * * \\
(0,008)\end{array}$ & $\begin{array}{l}-0,531 \\
(0,201)\end{array}$ & $\begin{array}{l}-0,450 \\
(0,310)\end{array}$ \\
\hline & Loja & $\begin{array}{c}-0,00367 * * \\
(0,047)\end{array}$ & $\begin{array}{l}-0,665 \\
(0,342)\end{array}$ & $\begin{array}{l}-0,714 \\
(0,337)\end{array}$ \\
\hline & Los Ríos & $\begin{array}{c}0,00455 \\
(0,174)\end{array}$ & $\begin{array}{l}-0,890 \\
(0,145)\end{array}$ & $\begin{array}{l}-0,838 \\
(0,203)\end{array}$ \\
\hline & Manabí & $\begin{array}{c}-0,00280 \\
(0,102)\end{array}$ & $\begin{array}{l}-0,547 \\
(0,152)\end{array}$ & $\begin{array}{l}-0,580 \\
(0,153)\end{array}$ \\
\hline & Napo & $\begin{array}{c}0,00312 \\
(0,653)\end{array}$ & $\begin{array}{c}0,497 \\
(0,268)\end{array}$ & $\begin{array}{c}0,541 \\
(0,251)\end{array}$ \\
\hline & Pastaza & $\begin{array}{c}0,00274 \\
(0,727)\end{array}$ & $\begin{array}{c}0,235 \\
(0,525)\end{array}$ & $\begin{array}{c}0,248 \\
(0,519)\end{array}$ \\
\hline & Tungurahua & $\begin{array}{c}0,000038 \\
(0,983)\end{array}$ & $\begin{array}{l}-0,279 \\
(0,372)\end{array}$ & $\begin{array}{l}-0,278 \\
(0,403)\end{array}$ \\
\hline
\end{tabular}

NOTAS: 1. p-values robustos entre paréntesis. 2. *** $\mathrm{p}<0,01$, ** $\mathrm{p}<0,05, * \mathrm{p}<0,1.3$. dy/dx para variables dummy representa el cambio que experimenta la probabilidad de invertir en I+D cuando la dummy correspondiente pasa de 0 a 1 . 
CUADRO 2 (continuación)

LAS DECISIONES DE INVERSIÓN Y DE GASTO EN I+D

\begin{tabular}{|c|c|c|c|c|}
\hline & \multirow[b]{2}{*}{ Variables } & \multirow{2}{*}{$\begin{array}{c}\text { Si/No I+D } \\
\text { y = Prob. pred } \\
(\mathrm{I}+\mathrm{D}=\mathbf{1})=\mathbf{0 , 0 0 9} \\
\text { dy/dx } \\
\text { (Efectos marg. medios) }\end{array}$} & \multicolumn{2}{|c|}{ Gasto en I+D } \\
\hline & & & Heckman & MCO \\
\hline \multirow[t]{4}{*}{$\begin{array}{l}\text { Ubicación geográfica de } \\
\text { la empresa }\end{array}$} & Zamora & $\begin{array}{c}0,00623 \\
(0,479)\end{array}$ & $\begin{array}{l}1,221 * * \\
(0,024)\end{array}$ & $\begin{array}{l}1,267 * * \\
(0,449)\end{array}$ \\
\hline & Sucumbíos & $\begin{array}{c}0,00441 \\
(0,523)\end{array}$ & $\begin{array}{l}-0,918 \\
(0,484)\end{array}$ & $\begin{array}{l}-0,881 \\
(0,531)\end{array}$ \\
\hline & Orellana & $\begin{array}{c}-0,00288 \\
(0,551)\end{array}$ & $\begin{array}{c}-0,911 * * \\
(0,022)\end{array}$ & $\begin{array}{c}-0,947 * * \\
(0,023)\end{array}$ \\
\hline & Santo Domingo & $\begin{array}{c}0,00258 \\
(0,376)\end{array}$ & $\begin{array}{c}0,104 \\
(0,722)\end{array}$ & $\begin{array}{c}0,134 \\
(0,666)\end{array}$ \\
\hline \multirow[t]{11}{*}{ Sector manufacturero } & Textiles & $\begin{array}{c}-0,00220 * \\
(0,053)\end{array}$ & $\begin{array}{l}-0,053 \\
(0,851)\end{array}$ & $\begin{array}{l}-0,085 \\
(0,777)\end{array}$ \\
\hline & Calzado y piel & $\begin{array}{c}-0,00142 \\
(0,483)\end{array}$ & $\begin{array}{c}0,382 \\
(0,435)\end{array}$ & $\begin{array}{c}0,367 \\
(0,480)\end{array}$ \\
\hline & Madera y papel & $\begin{array}{c}-0,00346^{* * *} \\
(0,004)\end{array}$ & $\begin{array}{c}0,282 \\
(0,516)\end{array}$ & $\begin{array}{c}0,229 \\
(0,617)\end{array}$ \\
\hline & Químico & $\begin{array}{c}0,00798 * * \\
(0,018) \\
\end{array}$ & $\begin{array}{c}0,208 \\
(0,455)\end{array}$ & $\begin{array}{c}0,286 \\
(0,322)\end{array}$ \\
\hline & Caucho y plástico & $\begin{array}{c}-0,00100 \\
(0,624)\end{array}$ & $\begin{array}{c}0,589 \\
(0,113) \\
\end{array}$ & $\begin{array}{c}0,578 \\
(0,146) \\
\end{array}$ \\
\hline & Prod. no metálicos & $\begin{array}{c}-0,00358 * * \\
(0,016)\end{array}$ & $\begin{array}{c}0,099 \\
(0,813)\end{array}$ & $\begin{array}{c}0,047 \\
(0,917)\end{array}$ \\
\hline & Muebles & $\begin{array}{c}-0,00223^{*} \\
(0,063)\end{array}$ & $\begin{array}{l}-0,068 \\
(0,837) \\
\end{array}$ & $\begin{array}{l}-0,098 \\
(0,783)\end{array}$ \\
\hline & Prod. metálicos & $\begin{array}{c}-0,00225^{*} \\
(0,069)\end{array}$ & $\begin{array}{c}0,440 \\
(0,229) \\
\end{array}$ & $\begin{array}{c}0,409 \\
(0,295) \\
\end{array}$ \\
\hline & Maq. ofic. y elect. & $\begin{array}{c}0,00722 * * \\
(0,038)\end{array}$ & $\begin{array}{c}0,556 \\
(0,161)\end{array}$ & $\begin{array}{c}0,634 \\
(0,126)\end{array}$ \\
\hline & Com./prec./méd & $\begin{array}{c}0,0115 * \\
(0,087)\end{array}$ & $\begin{array}{l}-0,417 \\
(0,400) \\
\end{array}$ & $\begin{array}{l}-0,308 \\
(0,554)\end{array}$ \\
\hline & Eq. de transporte & $\begin{array}{c}-0,00301 \\
(0,191)\end{array}$ & $\begin{array}{c}0,245 \\
(0,683)\end{array}$ & $\begin{array}{c}0,205 \\
(0,747)\end{array}$ \\
\hline
\end{tabular}

NOTAS: 1. $p$-values robustos entre paréntesis. 2. $* * * p<0,01, * * p<0,05, * p<0,1$. 3. dy/dx para variables dummy representa el cambio que experimenta la probabilidad de invertir en I+D cuando la dummy correspondiente pasa de 0 a 1. 
CUADRO 2 (continuación)

LAS DECISIONES DE INVERSIÓN Y DE GASTO EN I+D

\begin{tabular}{|c|c|c|c|c|}
\hline & \multirow[b]{2}{*}{ Variables } & \multirow{2}{*}{$\begin{array}{c}\text { Si/No I+D } \\
\text { y = Prob. pred } \\
(\mathbf{I}+\mathbf{D}=\mathbf{1})=\mathbf{0 , 0 0 9} \\
\text { dy/dx } \\
\text { (Efectos marg. medios) }\end{array}$} & \multicolumn{2}{|c|}{ Gasto en I+D } \\
\hline & & & Heckman & MCO \\
\hline \multirow[t]{4}{*}{$\begin{array}{l}\text { Forma jurídica de la } \\
\text { empresa }\end{array}$} & Empresa privada & $\begin{array}{c}-0,000891 \\
(0,503) \\
\end{array}$ & $\begin{array}{c}0,568 * * * \\
(0,009)\end{array}$ & $\begin{array}{c}0,559 * * \\
(0,017)\end{array}$ \\
\hline & Empresa pública & $\begin{array}{l}0,0165 \\
(0,178)\end{array}$ & $\begin{array}{c}0,642 \\
(0,502)\end{array}$ & $\begin{array}{c}0,767 \\
(0,448)\end{array}$ \\
\hline & Cooperativa & $\begin{array}{c}0,000366 \\
(0,969)\end{array}$ & $\begin{array}{l}-0,590 \\
(0,459)\end{array}$ & $\begin{array}{l}-0,590 \\
(0,484)\end{array}$ \\
\hline & Asociación & $\begin{array}{c}0,00205 \\
(0,700) \\
\end{array}$ & $\begin{array}{c}0,351 \\
(0,404) \\
\end{array}$ & $\begin{array}{c}0,379 \\
(0,387) \\
\end{array}$ \\
\hline \multirow[t]{8}{*}{$\begin{array}{l}\text { Profesionalización de la } \\
\text { empresa }\end{array}$} & Red empresarial & $\begin{array}{c}0,00321 * * * \\
(0,009)\end{array}$ & $\begin{array}{l}0,392 * \\
(0,061)\end{array}$ & $\begin{array}{l}0,431 * \\
(0,054)\end{array}$ \\
\hline & Inv. de mercado & $\begin{array}{c}0,0228 * * * \\
(0,000)\end{array}$ & $\begin{array}{c}0,107 \\
(0,592)\end{array}$ & $\begin{array}{c}0,269 \\
(0,154)\end{array}$ \\
\hline & Contabilidad & $\begin{array}{c}0,00574 * * * \\
(0,001)\end{array}$ & $\begin{array}{c}0,878 * * * \\
(0,000) \\
\end{array}$ & $\begin{array}{c}0,949 * * * \\
(0,000)\end{array}$ \\
\hline & Financiamiento & $\begin{array}{c}0,00335 * * * \\
(0,000)\end{array}$ & $\begin{array}{l}-0,032 \\
(0,852)\end{array}$ & $\begin{array}{c}0,009 \\
(0,961) \\
\end{array}$ \\
\hline & Medio ambiente & $\begin{array}{c}0,0103 * * * \\
(0,000) \\
\end{array}$ & $\begin{array}{l}-0,053 \\
(0,787) \\
\end{array}$ & $\begin{array}{c}0,039 \\
(0,834) \\
\end{array}$ \\
\hline & Formación & $\begin{array}{c}0,0188 * * * \\
(0,000)\end{array}$ & $\begin{array}{c}0,047 \\
(0,831)\end{array}$ & $\begin{array}{c}0,207 \\
(0,298)\end{array}$ \\
\hline & TIC & $\begin{array}{c}0,0120 * * * \\
(0,000)\end{array}$ & $\begin{array}{c}0,447 \\
(0,118) \\
\end{array}$ & $\begin{array}{c}0,585 * * \\
(0,033)\end{array}$ \\
\hline & $\begin{array}{l}\text { Principal cliente } \\
\text { ext. }\end{array}$ & $\begin{array}{c}0,00216 \\
(0,398)\end{array}$ & $\begin{array}{c}0,035 \\
(0,916)\end{array}$ & $\begin{array}{l}0,060 \\
(0,864\end{array}$ \\
\hline
\end{tabular}

NOTAS: 1.p-values robustos entre paréntesis. 2. *** $p<0,01, * * \mathrm{p}<0,05, * p<0,1$. 3. dy/dx para variables dummy representa el cambio que experimenta la probabilidad de invertir en I+D cuando la dummy correspondiente pasa de 0 a 1. 


\section{CUADRO 2 (continuación)}

LAS DECISIONES DE INVERSIÓN Y DE GASTO EN I+D

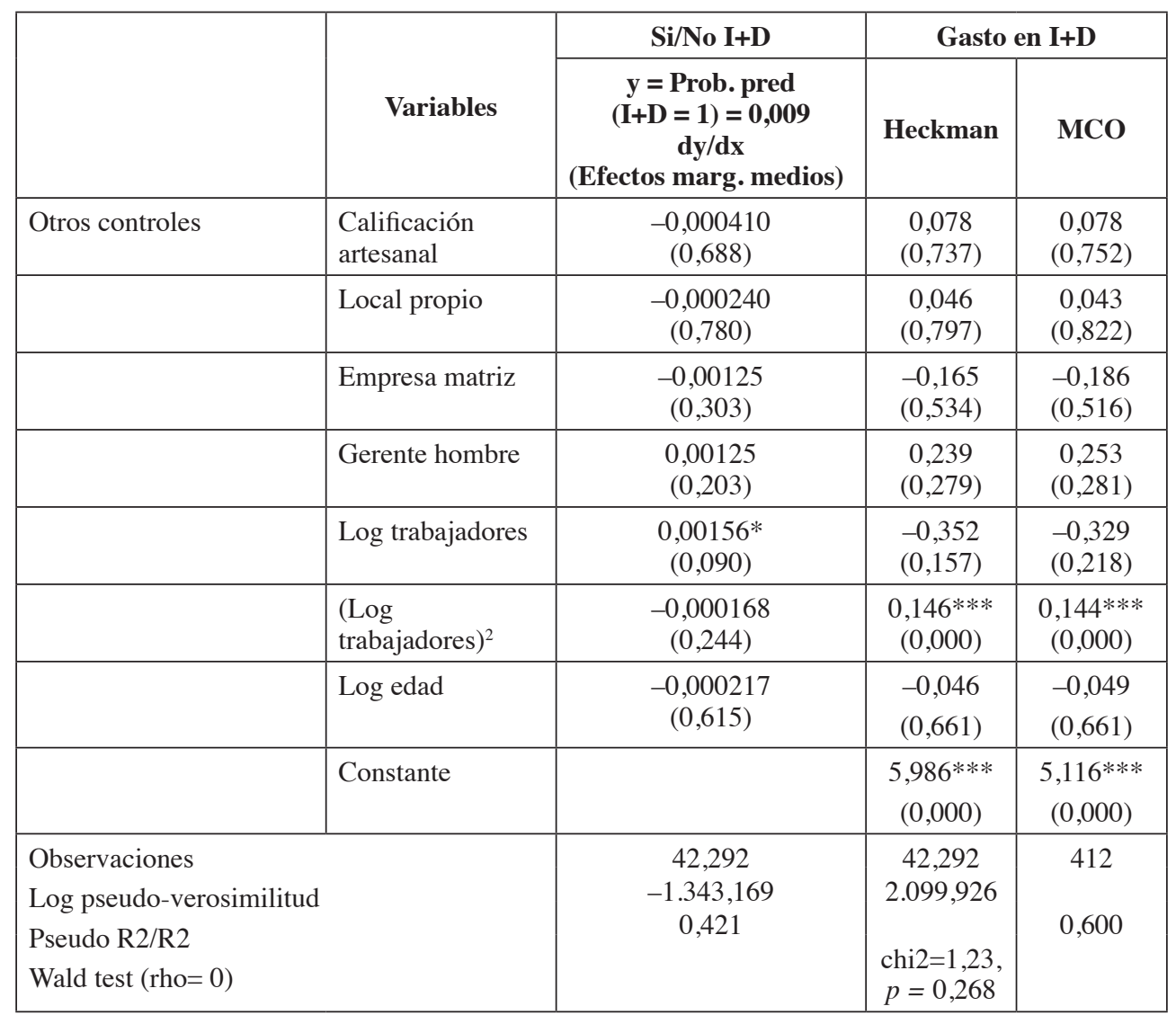

NOTAS: 1.p-values robustos entre paréntesis. 2. *** $\mathrm{p}<0,01$, ** $\mathrm{p}<0,05, * \mathrm{p}<0,1.3$. dy/dx para variables dummy representa el cambio que experimenta la probabilidad de invertir en I+D cuando la dummy correspondiente pasa de 0 a 1. 


\subsection{Metodología de estimación y resultados del modelo de regresión para el gasto en $I+D$}

Para estimar una ecuación de gasto en $\mathrm{I}+\mathrm{D}$ aplicable a todas las empresas (independientemente de que inviertan o no en I+D) se utilizará el método de selección de muestra de Heckman (1979). Este método de estimación resulta en principio conveniente debido a que hay en los datos muchas empresas que no invierten en $\mathrm{I}+\mathrm{D}$, lo que nos lleva a observar muchos «0» en los datos de gasto en I+D. Cuando la variable dependiente se presenta de esta forma, se la conoce como censurada ${ }^{9}$. Por medio de la estimación de este modelo se podrá contrastar si existe o no sesgo por selección. Si dicho sesgo no existiera se optará por el método más eficiente de regresión por Mínimos Cuadrados Ordinarios.

Según el método de Heckman para corregir por problemas de selección de muestra, el modelo a estimar va a constar de dos ecuaciones: la primera involucra la decisión de hacer o no I+D (parte de elección discreta binaria del modelo), y la segunda es la ecuación de regresión (ecuación de interés) en la que la variable dependiente es el gasto en I+D (transformado en logaritmos) procedente de las empresas que invierten en I+D. La primera de las ecuaciones se corresponde con la ecuación [1] anteriormente estimada en la sección 3.1. La segunda es la siguiente:

$$
G_{-} I+D_{i}=\gamma_{0}+x_{i}^{\prime} \gamma_{1}+u_{i}
$$

únicamente observada para las empresas que realizan un gasto positivo en $\mathrm{I}+\mathrm{D}$ $\left(G_{-} I+D_{i}>0\right)$. Las variables explicativas en la ecuación [6] coinciden con las de la ecuación [1].

Un supuesto importante en el modelo de regresión de Heckman es que los errores de las dos ecuaciones implicadas $\left(\varepsilon_{i} u_{i}\right)$ siguen una distribución Normal con media 0 , desviación estándar $\sigma_{\varepsilon}$ y 1 , respectivamente, y donde un parámetro denominado $\rho$ representa la correlación entre ambos términos de error. Si el parámetro $\rho$ resulta estadísticamente no significativo, no existe correlación entre las dos ecuaciones y podemos estimar simplemente la ecuación [6] por Mínimos Cuadrados Ordinarios con las observaciones de empresas que tienen un gasto positivo en $\mathrm{I}+\mathrm{D}$ (en nuestro caso 412 empresas).

Los resultados de la estimación de la ecuación de gasto en $\mathrm{I}+\mathrm{D}$ de las empresas por medio del modelo de regresión de Heckman se muestran en la segunda columna del Cuadro 2. Asimismo, al final de esta columna se muestra el contraste realizado de la hipótesis nula $\rho=0$, hipótesis que no puede ser rechazada ( $p$-value $=0,268$ ). Por lo tanto, se constata que la ecuación de gasto en I+D no presenta problemas de

\footnotetext{
${ }^{9}$ Otro de los métodos utilizados para solucionar en la estimación este problema de variable dependiente censurada es el modelo Tobit (TOBIN, 1958). Sin embargo, se trata de un método más restrictivo debido a que impone, por ejemplo, que el signo del efecto de las variables explicativas sea el mismo tanto a la hora de explicar la decisión implicada (en nuestro caso la decisión de realizar o no I+D) como la variable continua de gasto en I+D.
} 
sesgo por selección de muestra y, por ello, es adecuada y más eficiente la estimación por medio del tradicional método de mínimos cuadrados ordinarios.

La tercera columna del Cuadro 2 presenta los resultados por Mínimos Cuadrados Ordinarios, que son bastante parejos a los obtenidos por el método de Heckman. Ello es consecuencia de la no necesidad estadística de corrección por selección de muestra en nuestro modelo. Las variables de ubicación geográfica que son positivas y estadísticamente significativas frente a la categoría de referencia (Pichincha) para explicar el gasto en I+D son Carchi y Zamora. En cambio, El Oro (al igual que en la decisión de invertir o no en I+D) y Orellana presentan un signo negativo y son estadísticamente significativas. En relación a los sectores, si bien ninguno resulta significativo en la ecuación del gasto en $\mathrm{I}+\mathrm{D}$, el sector de Maquinaria de OficinaElectrónica y Otra Maquinaria Especializada se mantiene muy cerca de mostrar un efecto positivo y significativo ( $p$-value $=0,126$ ), siendo este un sector que ya había mostrado un efecto positivo y significativo en la decisión de invertir o no en I+D. La única forma jurídica que está asociada positivamente al gasto en I+D de las empresas es la categoría de Empresa Privada. Es interesante resaltar también de los resultados de esta tabla que pertenecer a una red empresarial, llevar contabilidad y haber implementado las TIC tiene un efecto significativo y positivo en la ecuación de gasto en I+D, al igual que ya lo tenía en la ecuación explicativa de la decisión de invertir o no en este tipo de actividades. Realizar estudios de investigación de mercado ha perdido su significatividad en la ecuación de gasto, a pesar de que su p-value está alrededor del 0,15. Las variables de financiación ajena, de inversión en actividades medioambientales o en actividades de formación del personal de la empresa reflejan que son relevantes para la decisión de inversión en I+D, pero su relevancia no se traslada a la ecuación de gasto en I+D (donde pierden su significatividad estadística). Por último, dentro de los otros controles incluidos en la estimación de la ecuación del gasto resulta significativa y positiva la variable de tamaño de la empresa, que ya lo era en la decisión dicotómica.

\subsection{Relación de la productividad de las empresas con el gasto en $I+D$}

En la literatura sobre productividad, la medición de esta variable puede llevarse a cabo de forma muy completa cuando se utilizan datos de panel, como es el caso de los métodos utilizados por Olley y Pakes (1996), Levinsohn y Petrin (2003), Máñez et al. (2009), entre otros. Sin embargo, en nuestro caso al disponer solo de un corte transversal, se deben buscar otras alternativas para obtener indicadores de productividad. En este trabajo se usará un primer indicador que es la productividad por trabajador, un segundo indicador que es el valor añadido por trabajador, y un tercer y cuarto indicadores que provienen de la estimación de una función de producción Cobb Douglas tradicional para determinar la Productividad Total de los Factores (PTF), tanto en su versión de producción como de Valor Añadido (PTF_VA). Todos estos indicadores aparecerán transformados en logaritmos en el momento de 
la estimación. Se debe tomar en consideración que en esta etapa de estimación la variable dependiente será la productividad en sus distintas versiones. En el presente trabajo la producción se mide a través de las ventas de cada empresa.

Para obtener los valores del primer indicador de productividad (productividad por trabajador, $\left.q_{i}\right)$ se calcula la ratio entre la producción total $\left(Q_{i}\right)$ y el número de trabajadores $\left(l_{i}\right)$ de la empresa:

$$
q_{i}=\frac{Q_{i}}{l_{i}}
$$

El segundo indicador de productividad es el valor añadido por trabajador $\left(v a_{i}\right)$, que se obtiene con la producción total de la empresa menos los gastos en materiales $\left(m_{i}\right)$, dividido entre el número de trabajadores:

$$
\begin{gathered}
V A_{i}=Q_{i}-m_{i} \\
v a_{i}=\frac{V A_{i}}{l_{i}}
\end{gathered}
$$

El tercer indicador de productividad es una medida de PTF que proviene de la estimación de una función de producción Cobb Douglas que incluye como inputs los gastos en materiales, el capital $\left(k_{i}\right)$ y el número de trabajadores ${ }^{10}$. En este caso se busca encontrar una predicción de productividad para cada empresa que será el residuo estimado $\left(\varepsilon_{Q i}\right)$ de la ecuación [10]. Este residuo estimado, al ser la parte de la función de producción que no ha podido ser explicada por las variables de input, es una aproximación a la productividad total asociada a los factores intrínsecos e inobservados de cada empresa. Debido a que se cuenta con información de corte transversal y, por lo tanto, solo se dispone de información de las variables para un período de tiempo, no se puede estimar la PTF por medio de métodos más sofisticados como los mencionados anteriormente. El método de estimación utilizado será el de $\mathrm{MCO}^{11}$.

$$
Q_{i}=\alpha+\beta_{m} m_{i}+\beta_{l} l_{i}+\beta_{k} k_{i}+\varepsilon_{Q i}
$$

El cuarto indicador consiste en una estimación de la PTF por medio del uso de la variable de valor añadido en lugar de producción en la función Cobb Douglas, lo que implica, por la propia definición de valor añadido (producción menos materiales),

\footnotetext{
${ }^{10}$ Las variables de la función de producción se usarán en logaritmos.

${ }^{11}$ El coeficiente para materiales $(\beta m)$ es de 0,58 , para trabajadores $(\beta l)$ de 0,40 y para el capital $(\beta k)$ de 0,12 , todos ellos significativos al 1 por 100. Los errores estándar se calculan robustos a heteroscedasticidad. No se rechaza la hipótesis nula de rendimientos constantes a escala.
} 
que los inputs para los que se estiman coeficientes según esta aproximación son solo el número de trabajadores y el capital ${ }^{12}$ :

$$
V A_{i}=\beta_{l} l_{i}+\beta_{k} k_{i}+\varepsilon_{V A_{i}}
$$

Una vez obtenidos los cuatro indicadores estos serán secuencialmente las variables dependientes del modelo. Las variables explicativas comunes a los cuatro indicadores de productividad serán el conjunto de variables dummy que caracterizan a las empresas (ubicación geográfica, sector manufacturero y forma jurídica), las variables de profesionalización de la empresa (investigación de mercado, formación, TIC y principal cliente extranjero), y la variable de edad de la empresa en logaritmos $\left(a_{i}\right)$. Por su parte, dado que las variables número de trabajadores y el capital ya han sido consideradas para estimar las ecuaciones [10] y [11] y obtener la PTF (PTF_ VA), las variables de número de trabajadores y la variable de intensidad de capital por trabajador no son incluidas como explicativas de los indicadores tres y cuatro (PTF con producción y PTF con valor añadido, respectivamente). Sin embargo, sí son consideradas como controles para las estimaciones que trabajan con los indicadores [7] y [9] (no se trata de medidas de PTF, se trata de productividad del trabajo calculada con producción y con valor añadido, respectivamente). Por último, en todas las estimaciones, independientemente del indicador de productividad considerado, se incluye como explicativa de interés el gasto en I+D. El objetivo del uso de los cuatro indicadores de productividad es la valoración de la robustez de los resultados sobre la relación de la productividad de las empresas con la inversión en I+D. A continuación se presentan las distintas especificaciones planteadas (por simplicidad se han omitido los coeficientes asociados a cada una de las explicativas, aunque debe entenderse que están implícitos):

$$
\begin{aligned}
& \widehat{q_{i}}=G_{-} I D_{i}+d p_{i}+d s_{i}+d j_{i}+d m_{i}+d f_{i}+d t_{i}+d e x_{i}+a_{i}+l_{i}+l_{i}^{2}+(k / l)_{i}+\varepsilon_{i} \\
& \widehat{v a_{i}}=G_{-} I D_{i}+d p_{i}+d s_{i}+d j_{i}+d m_{i}+d f_{i}+d t_{i}+d e x_{i}+a_{i}+l_{i}+l_{i}^{2}+(k / l)_{i}+\varepsilon_{i} \\
& \widehat{\varepsilon_{Q_{i}}}=G_{-} I D_{i}+d p_{i}+d s_{i}+d j_{i}+d m_{i}+d f_{i}+d t_{i}+d e x_{i}+a_{i}+\varepsilon_{i} \\
& \widehat{\varepsilon_{V A_{i}}}=G_{-} I D_{i}+d p_{i}+d s_{i}+d j_{i}+d m_{i}+d f_{i}+d t_{i}+\operatorname{dex}_{i}+a_{i}+\varepsilon_{i}
\end{aligned}
$$

En el Cuadro 3 se muestran los resultados de las regresiones con cada uno de los cuatro indicadores de productividad como variable dependiente. Nuestra principal variable de interés en estas estimaciones es la que indica si es o no significativa la relación entre el gasto de I+D de las empresas y su productividad. En las estimaciones, junto con la variable de gasto aparece un residuo predicho que proviene de la etapa previa de estimación en este trabajo, donde por medio de MCO se estimó el gasto en I+D de las empresas. De esta ecuación de gasto se ha obtenido el residuo predicho

${ }^{12}$ El coeficiente para trabajadores $(\beta l)$ es de 0,93 y para el capital $(\beta k)$ de 0,31 , ambos significativos al 1 por 100 . 
que se incluye ahora en las regresiones de productividad. El objetivo es que nos permita contrastar y corregir el potencial problema de endogeneidad que el gasto en I+D puede presentar en una ecuación de productividad. Los resultados del Cuadro 3 confirman tanto la conveniencia de esta corrección (el residuo de la ecuación del gasto en I+D es estadísticamente significativo) como que independientemente de la medida de productividad utilizada se obtiene una relación positiva y significativa con el gasto en I+D de las empresas (si bien el efecto es más débil con indicadores de productividad basados en la PTF).

Dado que la variable dependiente está en logaritmos en las cuatro regresiones, la interpretación de los coeficientes asociados a variables explicativas en logaritmos es la de una elasticidad. Así, por ejemplo, se obtiene que si el gasto en I+D de una empresa aumenta en un 1 por 100, su productividad por trabajador aumenta en un 0,316 por 100 (y su productividad total de los factores en un 0,018 por 100).

En cuanto a la ubicación geográfica de la empresa, casi todas las ubicaciones presentan una menor productividad que la categoría de referencia, Pichincha. Como excepciones se encuentran Galápagos y Orellana que justifican una mayor productividad con cualquiera de los cuatro indicadores utilizados. También la mayoría de sectores presentan menores niveles de productividad que el sector de referencia, Alimentación-Bebidas y Tabaco, con la excepción del sector químico que está asociado a los mayores niveles de productividad. En lo referente a la forma jurídica de la empresa, la que presenta mayores niveles de productividad es la empresa privada y las que presentan menores niveles son las empresas sin fines de lucro, las asociaciones y las cooperativas de ahorro y de crédito (que realizan actividades manufactureras). La categoría de referencia en este caso eran las empresas adscritas a personas naturales.

Para las actividades consideradas de profesionalización de la empresa que además aparecen en la literatura como posiblemente ligadas a la mejora de su productividad, es decir, si la empresa invierte en investigación de mercado, en actividades de formación de su mano de obra, si hace uso de las TIC y si exporta (esta última variable aproximada en nuestro caso por la variable de si el principal cliente de la empresa es extranjero), se obtienen los siguientes resultados: la variable más relevante parece ser el uso de las TIC, con signo positivo y altamente significativo sobre todos los indicadores de productividad; le sigue la variable de principal cliente extranjero, aunque esta resulta únicamente significativa (y positiva) con los dos indicadores sencillos de productividad que no se basan en medidas de la PTF; el resto de variables no son estadísticamente significativas.

Dado que la interpretación de los coeficientes asociados a variables dummy tiene interpretación de semielasticidad (al estar las variables dependientes en logaritmos), se obtiene, a modo de ejemplo, que si una empresa pasara de no utilizar a utilizar TIC, obtendría un aumento de un 15,4 por 100 en su productividad por trabajador (y de un 7,4 por 100 en su productividad total de los factores).

Por último, la edad de la empresa solo resulta significativa (y positiva) para los dos indicadores sencillos de productividad, y el número de trabajadores de la empresa (con cierta curvatura) y el capital por trabajador también son positivos y significativos para dichos indicadores. 


\section{CUADRO 3 \\ RELACIÓN DE LA PRODUCTIVIDAD DE LAS EMPRESAS CON EL GASTO EN I+D}

\begin{tabular}{|c|c|c|c|c|}
\hline & (1) & (2) & (3) & (4) \\
\hline Variables & $\begin{array}{c}\text { Log } \\
\text { Productividad } \\
\text { por } \\
\text { Trabajador }\end{array}$ & $\begin{array}{l}\text { Log VA por } \\
\text { Trabajador }\end{array}$ & PTF & PTF_VA \\
\hline $\log$ gasto $\mathrm{I}+\mathrm{D}$ & $\begin{array}{c}0,316 * * * \\
(0,000)\end{array}$ & $\begin{array}{c}0,277 * * * \\
(0,000)\end{array}$ & $\begin{array}{l}0,018 * \\
(0,100)\end{array}$ & $\begin{array}{c}0,044 * * \\
(0,015)\end{array}$ \\
\hline residuo_gasto $\mathrm{I}+\mathrm{D}^{3}$ & $\begin{array}{c}-0,316 * * * \\
(0,000)\end{array}$ & $\begin{array}{c}-0,271 * * * \\
(0,000)\end{array}$ & $\begin{array}{c}-0,020 * \\
(0,053)\end{array}$ & $\begin{array}{c}-0,047 * * * \\
(0,003)\end{array}$ \\
\hline Azuay & $\begin{array}{c}-0,285 * * * \\
(0,000)\end{array}$ & $\begin{array}{c}-0,326 * * * \\
(0,000)\end{array}$ & $\begin{array}{c}-0,155 * * * \\
(0,000)\end{array}$ & $\begin{array}{c}-0,269 * * * \\
(0,000)\end{array}$ \\
\hline Bolívar & $\begin{array}{c}-0,583 * * * \\
(0,000)\end{array}$ & $\begin{array}{c}-0,561 * * * \\
(0,000)\end{array}$ & $\begin{array}{c}-0,155 * * * \\
(0,000)\end{array}$ & $\begin{array}{c}-0,391 * * * \\
(0,000)\end{array}$ \\
\hline Cañar & $\begin{array}{c}-0,097 * * * \\
(0,007)\end{array}$ & $\begin{array}{c}-0,189 * * * \\
(0,000)\end{array}$ & $\begin{array}{c}-0,181 * * * \\
(0,000)\end{array}$ & $\begin{array}{c}-0,386 * * * \\
(0,000)\end{array}$ \\
\hline Carchi & $\begin{array}{c}-0,677 * * * \\
(0,000)\end{array}$ & $\begin{array}{c}-0,715 * * * \\
(0,000)\end{array}$ & $\begin{array}{c}-0,194 * * * \\
(0,000)\end{array}$ & $\begin{array}{c}-0,351 * * * \\
(0,000)\end{array}$ \\
\hline Cotopaxi & $\begin{array}{c}-0,084 * * * \\
(0,002)\end{array}$ & $\begin{array}{c}-0,234 * * * \\
(0,000)\end{array}$ & $\begin{array}{c}-0,183 * * * \\
(0,000)\end{array}$ & $\begin{array}{c}-0,334 * * * \\
(0,000)\end{array}$ \\
\hline Chimborazo & $\begin{array}{c}-0,149 * * * \\
(0,000)\end{array}$ & $\begin{array}{c}-0,292 * * * \\
(0,000)\end{array}$ & $\begin{array}{c}-0,218 * * * \\
(0,000)\end{array}$ & $\begin{array}{c}-0,402 * * * \\
(0,000)\end{array}$ \\
\hline El Oro & $\begin{array}{c}0,440 * * * \\
(0,000)\end{array}$ & $\begin{array}{c}0,318 * * * \\
(0,000)\end{array}$ & $\begin{array}{l}-0,012 \\
(0,605) \\
\end{array}$ & $\begin{array}{l}-0,017 \\
(0,645) \\
\end{array}$ \\
\hline Esmeraldas & $\begin{array}{c}-0,386 * * * \\
(0,000)\end{array}$ & $\begin{array}{c}-0,385^{* * * *} \\
(0,000)\end{array}$ & $\begin{array}{c}-0,086 * * * \\
(0,001)\end{array}$ & $\begin{array}{c}-0,259 * * * \\
(0,000)\end{array}$ \\
\hline Guayas & $\begin{array}{c}-0,026 * * \\
(0,037)\end{array}$ & $\begin{array}{c}-0,061 * * * \\
(0,000)\end{array}$ & $\begin{array}{c}-0,044 * * * \\
(0,000)\end{array}$ & $\begin{array}{c}-0,046^{* * * *} \\
(0,003)\end{array}$ \\
\hline Imbabura & $\begin{array}{l}-0,032 \\
(0,184)\end{array}$ & $\begin{array}{c}-0,191 * * * \\
(0,000)\end{array}$ & $\begin{array}{c}-0,190 * * * \\
(0,000)\end{array}$ & $\begin{array}{c}-0,275^{* * * *} \\
(0,000)\end{array}$ \\
\hline Loja & $\begin{array}{c}0,122 * * * \\
(0,000)\end{array}$ & $\begin{array}{l}-0,003 \\
(0,932)\end{array}$ & $\begin{array}{c}-0,116^{* * * *} \\
(0,000)\end{array}$ & $\begin{array}{c}-0,140 * * * \\
(0,000)\end{array}$ \\
\hline Los Ríos & $\begin{array}{c}0,116 * * * \\
(0,000)\end{array}$ & $\begin{array}{l}0,067 * \\
(0,052)\end{array}$ & $\begin{array}{l}-0,015 \\
(0,477)\end{array}$ & $\begin{array}{c}-0,069 * \\
(0,055)\end{array}$ \\
\hline
\end{tabular}

NOTAS: 1. $p$-values robustos y obtenidos por bootstrapping entre paréntesis. 2 . *** $p<0,01, * * p<0,05$, $* p<0,1$. 3. Término que sirve para contrastar y corregir la endogeneidad del gasto en I+D en la ecuación de productividad. 4. Dado que la variable dependiente está en logaritmos, la interpretación de los coeficientes asociados a variables explicativas en logaritmos es la de una elasticidad. La de los coeficientes asociados a variables dummy tiene interpretación de semielasticidad. 
CUADRO 3 (continuación)

RELACIÓN DE LA PRODUCTIVIDAD DE LAS EMPRESAS CON EL GASTO EN I+D

\begin{tabular}{|c|c|c|c|c|}
\hline & (1) & (2) & (3) & (4) \\
\hline Variables & $\begin{array}{c}\text { Log } \\
\text { Productividad } \\
\text { por } \\
\text { Trabajador }\end{array}$ & $\begin{array}{l}\text { Log VA por } \\
\text { Trabajador }\end{array}$ & PTF & PTF_VA \\
\hline Manabí & $\begin{array}{c}-0,085^{* * *} * \\
(0,000)\end{array}$ & $\begin{array}{c}-0,226 * * * \\
(0,000) \\
\end{array}$ & $\begin{array}{c}-0,168 * * * \\
(0,000)\end{array}$ & $\begin{array}{c}-0,324 * * * \\
(0,000)\end{array}$ \\
\hline Morona Santiago & $\begin{array}{c}-0,131 \text { *** } \\
(0,001)\end{array}$ & $\begin{array}{c}-0,201 * * * \\
(0,000)\end{array}$ & $\begin{array}{c}-0,132 * * * \\
(0,000)\end{array}$ & $\begin{array}{c}-0,198 * * * \\
(0,000)\end{array}$ \\
\hline Napo & $\begin{array}{c}-0,192 * * * \\
(0,002)\end{array}$ & $\begin{array}{c}-0,184 * * * \\
(0,004)\end{array}$ & $\begin{array}{c}-0,082 * * \\
(0,041)\end{array}$ & $\begin{array}{l}-0,065 \\
(0,301)\end{array}$ \\
\hline Pastaza & $\begin{array}{c}-0,283 * * * \\
(0,000)\end{array}$ & $\begin{array}{c}-0,375 * * * \\
(0,000)\end{array}$ & $\begin{array}{c}-0,195 * * * \\
(0,000)\end{array}$ & $\begin{array}{c}-0,318 * * * \\
(0,000)\end{array}$ \\
\hline Tungurahua & $\begin{array}{c}-0,071 * * * \\
(0,000)\end{array}$ & $\begin{array}{c}-0,232 * * * \\
(0,000)\end{array}$ & $\begin{array}{c}-0,192 * * * \\
(0,000)\end{array}$ & $\begin{array}{c}-0,315 * * * \\
(0,000)\end{array}$ \\
\hline Zamora & $\begin{array}{c}-0,599 * * * \\
(0,000)\end{array}$ & $\begin{array}{c}-0,680 * * * \\
(0,000)\end{array}$ & $\begin{array}{c}-0,271 * * * \\
(0,000)\end{array}$ & $\begin{array}{c}-0,414 * * * \\
(0,000)\end{array}$ \\
\hline Galápagos & $\begin{array}{c}0,350 * * * \\
(0,002) \\
\end{array}$ & $\begin{array}{c}0,400 * * * \\
(0,002) \\
\end{array}$ & $\begin{array}{c}0,275^{* * * *} \\
(0,001) \\
\end{array}$ & $\begin{array}{c}0,427 * * * \\
(0,001) \\
\end{array}$ \\
\hline Sucumbíos & $\begin{array}{l}0,100 * \\
(0,058)\end{array}$ & $\begin{array}{c}0,035 \\
(0,575) \\
\end{array}$ & $\begin{array}{c}-0,089 * * \\
(0,014)\end{array}$ & $\begin{array}{c}-0,175 * * * \\
(0,004)\end{array}$ \\
\hline Orellana & $\begin{array}{c}0,406^{* * *} * \\
(0,000)\end{array}$ & $\begin{array}{c}0,365 * * * \\
(0,000)\end{array}$ & $\begin{array}{c}0,048 \\
(0,244)\end{array}$ & $\begin{array}{c}0,149 * * \\
(0,025)\end{array}$ \\
\hline Santo Domingo & $\begin{array}{c}-0,062 * * \\
(0,017) \\
\end{array}$ & $\begin{array}{c}-0,058^{*} \\
(0,066) \\
\end{array}$ & $\begin{array}{r}-0,007 \\
(0,696) \\
\end{array}$ & $\begin{array}{l}-0,016 \\
(0,582) \\
\end{array}$ \\
\hline Santa Elena & $\begin{array}{c}-0,230 * * * \\
(0,000)\end{array}$ & $\begin{array}{c}-0,332 * * * \\
(0,000)\end{array}$ & $\begin{array}{c}-0,188^{* * * *} \\
(0,000)\end{array}$ & $\begin{array}{c}-0,295 * * * \\
(0,000)\end{array}$ \\
\hline Zonas no delimitadas & $\begin{array}{l}-0,279 \\
(0,204) \\
\end{array}$ & $\begin{array}{c}-0,478^{*} \\
(0,089) \\
\end{array}$ & $\begin{array}{r}-0,205 \\
(0,156) \\
\end{array}$ & $\begin{array}{l}-0,450 \\
(0,127) \\
\end{array}$ \\
\hline Textiles & $\begin{array}{c}-0,546 * * * \\
(0,000) \\
\end{array}$ & $\begin{array}{c}-0,329 * * * \\
(0,000) \\
\end{array}$ & $\begin{array}{c}0,025^{* * *} * \\
(0,000) \\
\end{array}$ & $\begin{array}{c}-0,269 * * * \\
(0,005) \\
\end{array}$ \\
\hline Calzado y piel & $\begin{array}{c}-0,311 \text { *** } \\
(0,000)\end{array}$ & $\begin{array}{c}-0,254 * * * \\
(0,000)\end{array}$ & $\begin{array}{c}-0,034^{*} \\
(0,000)\end{array}$ & $\begin{array}{c}-0,111 * * * \\
(0,072)\end{array}$ \\
\hline
\end{tabular}

NOTAS: 1. p-values robustos y obtenidos por bootstrapping entre paréntesis. 2. *** $p<0,01$, ** $p<0,05$ $* p<0,1$. 3. Término que sirve para contrastar y corregir la endogeneidad del gasto en I+D en la ecuación de productividad. 4. Dado que la variable dependiente está en logaritmos, la interpretación de los coeficientes asociados a variables explicativas en logaritmos es la de una elasticidad. La de los coeficientes asociados a variables dummy tiene interpretación de semielasticidad. 


\section{CUADRO 3 (continuación) \\ RELACIÓN DE LA PRODUCTIVIDAD DE LAS EMPRESAS CON EL GASTO EN I+D}

\begin{tabular}{|c|c|c|c|c|}
\hline & (1) & $(2)$ & (3) & (4) \\
\hline Variables & $\begin{array}{c}\text { Log } \\
\text { Productividad } \\
\text { por } \\
\text { Trabajador }\end{array}$ & $\begin{array}{l}\text { Log VA por } \\
\text { Trabajador }\end{array}$ & PTF & PTF_VA \\
\hline Madera y papel & $\begin{array}{c}-0,519 * * * \\
(0,000)\end{array}$ & $\begin{array}{c}-0,381 * * * \\
(0,000)\end{array}$ & $\begin{array}{c}-0,074 * * * \\
(0,000)\end{array}$ & $\begin{array}{c}-0,268 * * * \\
(0,000)\end{array}$ \\
\hline Químico & $\begin{array}{c}0,026 \\
(0,685)\end{array}$ & $\begin{array}{c}0,177 * * \\
(0,015)\end{array}$ & $\begin{array}{c}0,224 * * * \\
(0,000)\end{array}$ & $\begin{array}{c}0,332 * * * \\
(0,000)\end{array}$ \\
\hline Caucho y plástico & $\begin{array}{c}-0,376^{* * *} * \\
(0,000) \\
\end{array}$ & $\begin{array}{c}-0,262 * * * \\
(0,000) \\
\end{array}$ & $\begin{array}{c}0,000 \\
(0,989) \\
\end{array}$ & $\begin{array}{c}-0,119 * * \\
(0,018) \\
\end{array}$ \\
\hline Prod. no metálicos & $\begin{array}{c}-0,112 * * * \\
(0,000)\end{array}$ & $\begin{array}{c}-0,072 * * * \\
(0,003)\end{array}$ & $\begin{array}{l}-0,001 \\
(0,931)\end{array}$ & $\begin{array}{r}-0,030 \\
(0,229)\end{array}$ \\
\hline Muebles & $\begin{array}{c}-0,272 * * * \\
(0,000) \\
\end{array}$ & $\begin{array}{c}-0,206^{* * * *} \\
(0,000) \\
\end{array}$ & $\begin{array}{c}-0,057 \text { **** } \\
(0,000) \\
\end{array}$ & $\begin{array}{c}-0,167 * * * \\
(0,000) \\
\end{array}$ \\
\hline Prod. metálicos & $\begin{array}{c}-0,352 * * * \\
(0,000)\end{array}$ & $\begin{array}{c}-0,299 * * * \\
(0,000)\end{array}$ & $\begin{array}{c}-0,047 * * * \\
(0,000)\end{array}$ & $\begin{array}{c}-0,123 * * * \\
(0,000) \\
\end{array}$ \\
\hline Maq. ofic. y elect. & $\begin{array}{c}-0,477 * * * \\
(0,000)\end{array}$ & $\begin{array}{c}-0,303^{* * *} \\
(0,000)\end{array}$ & $\begin{array}{l}-0,004 \\
(0,882)\end{array}$ & $\begin{array}{c}-0,165 * * * \\
(0,000)\end{array}$ \\
\hline Com./prec./méd & $\begin{array}{c}-0,333 * * * \\
(0,000)\end{array}$ & $\begin{array}{l}-0,105 \\
(0,128)\end{array}$ & $\begin{array}{c}0,061 \\
(0,125)\end{array}$ & $\begin{array}{l}-0,052 \\
(0,424)\end{array}$ \\
\hline Eq. de transporte & $\begin{array}{c}-0,206 * * * \\
(0,000) \\
\end{array}$ & $\begin{array}{c}-0,120 * * \\
(0,024) \\
\end{array}$ & $\begin{array}{c}0,021 \\
(0,514) \\
\end{array}$ & $\begin{array}{l}-0,077 \\
(0,124) \\
\end{array}$ \\
\hline Empresa sin fines de lucro & $\begin{array}{c}-0,475^{*} \\
(0,065)\end{array}$ & $\begin{array}{c}-0,635^{*} \\
(0,075)\end{array}$ & $\begin{array}{c}-0,366^{* * *} \\
(0,009)\end{array}$ & $\begin{array}{c}-0,837 * * \\
(0,022)\end{array}$ \\
\hline Empresa privada & $\begin{array}{c}0,215 * * * \\
(0,000) \\
\end{array}$ & $\begin{array}{c}0,256^{* * *} * \\
(0,000) \\
\end{array}$ & $\begin{array}{c}0,092 * * * \\
(0,003) \\
\end{array}$ & $\begin{array}{c}0,159 * * * \\
(0,001) \\
\end{array}$ \\
\hline Empresa de control ext. & $\begin{array}{c}1,045 \\
(0,150) \\
\end{array}$ & $\begin{array}{c}0,886 \\
(0,255) \\
\end{array}$ & $\begin{array}{c}0,134 \\
(0,702) \\
\end{array}$ & $\begin{array}{c}0,680 \\
(0,445) \\
\end{array}$ \\
\hline Empresa pública & $\begin{array}{l}-0,283 \\
(0,209) \\
\end{array}$ & $\begin{array}{l}-0,054 \\
(0,822) \\
\end{array}$ & $\begin{array}{c}0,371 \\
(0,134) \\
\end{array}$ & $\begin{array}{l}-0,052 \\
(0,802) \\
\end{array}$ \\
\hline Empresa de gobierno local & $\begin{array}{l}-0,940 \\
(0,180)\end{array}$ & $\begin{array}{c}-0,874 \\
(0,334)\end{array}$ & $\begin{array}{c}0,314 \\
(0,650)\end{array}$ & $\begin{array}{r}-0,974 \\
(0,264)\end{array}$ \\
\hline
\end{tabular}

NOTAS: 1. p-values robustos y obtenidos por bootstrapping entre paréntesis. 2 . *** $p<0,01, * * p<0,05$, $* p<0,1$. 3. Término que sirve para contrastar y corregir la endogeneidad del gasto en I+D en la ecuación de productividad. 4. Dado que la variable dependiente está en logaritmos, la interpretación de los coeficientes asociados a variables explicativas en logaritmos es la de una elasticidad. La de los coeficientes asociados a variables dummy tiene interpretación de semielasticidad. 
CUADRO 3 (continuación)

RELACIÓN DE LA PRODUCTIVIDAD DE LAS EMPRESAS CON EL GASTO EN I+D

\begin{tabular}{|c|c|c|c|c|}
\hline & (1) & (2) & (3) & (4) \\
\hline Variables & $\begin{array}{c}\text { Log } \\
\text { Productividad } \\
\text { por } \\
\text { Trabajador }\end{array}$ & $\begin{array}{l}\text { Log VA por } \\
\text { Trabajador }\end{array}$ & PTF & PTF_VA \\
\hline Cooperativa de aho. y créd. & $\begin{array}{c}-0,217 * * \\
(0,023)\end{array}$ & $\begin{array}{c}-0,656 * * * \\
(0,000)\end{array}$ & $\begin{array}{c}-0,678 * * * \\
(0,000)\end{array}$ & $\begin{array}{c}-1,013 * * * \\
(0,000)\end{array}$ \\
\hline Cooperativa & $\begin{array}{c}0,007 \\
(0,984)\end{array}$ & $\begin{array}{c}0,106 \\
(0,765)\end{array}$ & $\begin{array}{c}0,102 \\
(0,678)\end{array}$ & $\begin{array}{l}-0,078 \\
(0,832)\end{array}$ \\
\hline Asociación & $\begin{array}{c}-0,343 * * \\
(0,034)\end{array}$ & $\begin{array}{c}-0,435 * * * \\
(0,009)\end{array}$ & $\begin{array}{l}-0,136 \\
(0,170)\end{array}$ & $\begin{array}{c}-0,390 * * \\
(0,021)\end{array}$ \\
\hline Inv. de Mercado & $\begin{array}{l}-0,024 \\
(0,465)\end{array}$ & $\begin{array}{l}-0,008 \\
(0,826)\end{array}$ & $\begin{array}{c}0,012 \\
(0,612)\end{array}$ & $\begin{array}{c}0,036 \\
(0,353)\end{array}$ \\
\hline Formación & $\begin{array}{c}0,020 \\
(0,473)\end{array}$ & $\begin{array}{c}0,014 \\
(0,648)\end{array}$ & $\begin{array}{l}-0,012 \\
(0,532)\end{array}$ & $\begin{array}{l}-0,011 \\
(0,734)\end{array}$ \\
\hline TIC & $\begin{array}{c}0,154 * * * \\
(0,000)\end{array}$ & $\begin{array}{c}0,184 * * * \\
(0,000)\end{array}$ & $\begin{array}{c}0,074 * * * \\
(0,000)\end{array}$ & $\begin{array}{c}0,176^{* * * *} \\
(0,000)\end{array}$ \\
\hline Principal cliente ext. & $\begin{array}{l}0,178 * * \\
(0,035)\end{array}$ & $\begin{array}{l}0,164 * * \\
(0,049)\end{array}$ & $\begin{array}{l}-0,006 \\
(0,906)\end{array}$ & $\begin{array}{c}0,017 \\
(0,852)\end{array}$ \\
\hline Log Trabajadores & $\begin{array}{c}0,082 * * * \\
(0,000)\end{array}$ & $\begin{array}{c}0,096 * * * \\
(0,000)\end{array}$ & & \\
\hline${\text { (Log Trabajadores })^{2}}^{2}$ & $\begin{array}{c}-0,030 * * * \\
(0,000)\end{array}$ & $\begin{array}{c}-0,029 * * * \\
(0,000)\end{array}$ & & \\
\hline Log edad & $\begin{array}{c}0,022 * * * \\
(0,000)\end{array}$ & $\begin{array}{c}0,026 * * * \\
(0,000)\end{array}$ & $\begin{array}{c}0,003 \\
(0,166)\end{array}$ & $\begin{array}{c}0,000 \\
(0,947)\end{array}$ \\
\hline Log capital por trab. & $\begin{array}{c}0,256 * * * \\
(0,000)\end{array}$ & $\begin{array}{c}0,246 * * * \\
(0,000)\end{array}$ & & \\
\hline Constante & $\begin{array}{c}5,596 * * * \\
(0,000)\end{array}$ & $\begin{array}{c}5,223 * * * \\
(0,000)\end{array}$ & $\begin{array}{c}0,026 \\
(0,635)\end{array}$ & $\begin{array}{c}0,002 \\
(0,985)\end{array}$ \\
\hline $\begin{array}{l}\text { Observaciones } \\
\text { R2 }\end{array}$ & $\begin{array}{c}40,358 \\
0,331\end{array}$ & $\begin{array}{c}40,358 \\
0,255\end{array}$ & $\begin{array}{c}40,358 \\
0,035\end{array}$ & $\begin{array}{c}40,358 \\
0,052\end{array}$ \\
\hline
\end{tabular}

NOTAS: 1. $p$-values robustos y obtenidos por bootstrapping entre paréntesis. 2 . *** $p<0,01$, ** $p<0,05$, $* p<0,1$. 3. Término que sirve para contrastar y corregir la endogeneidad del gasto en I+D en la ecuación de productividad. 4. Dado que la variable dependiente está en logaritmos, la interpretación de los coeficientes asociados a variables explicativas en logaritmos es la de una elasticidad. La de los coeficientes asociados a variables dummy tiene interpretación de semielasticidad. 


\section{Conclusiones}

El objetivo principal de este trabajo es investigar la relación entre el gasto de I+D de las empresas y su productividad. Como pasos intermedios a este objetivo principal, se han estimado tanto la probabilidad de invertir en I+D como la cuantía del gasto destinado por las empresas a estas actividades. En la estimación de las distintas etapas se han introducido, además de las variables explicativas de interés, una serie de controles tanto geográficos de ubicación de las empresas como sectoriales y referentes a su forma jurídica, obteniéndose en general que las empresas ubicadas en la provincia de Pichincha tienen una mayor propensión a invertir en I+D, realizan un mayor gasto en esta actividad, y también obtienen una mayor productividad. Asimismo, las empresas del sector químico son las que presentan una mayor probabilidad de invertir en I+D y las que también obtienen mayores productividades. Por último, la forma jurídica que tanto explica una mayor inversión en I+D como una mayor productividad es la de empresa privada.

Por lo que respecta a las variables de profesionalización de la empresa, las que resultan relevantes y positivas tanto para la decisión como para el gasto en I+D son la pertenencia a una red empresarial, que la empresa lleve contabilidad y que haga uso de las TIC. Otras variables de este ámbito tan solo son relevantes para justificar una mayor probabilidad de que la empresa invierta en $\mathrm{I}+\mathrm{D}$, como son las inversiones en investigación de mercado, en actividades medioambientales y en formación de la mano de obra, o que la empresa tenga capacidad para obtener financiamiento externo.

Por último, en las regresiones relativas a la productividad, más allá de los controles introducidos, estamos interesados en establecer el signo y la significatividad de la variable de gasto en I+D. A este respecto se obtiene que para cualquiera de los cuatro indicadores de productividad el signo estimado para esta variable es positivo y significativo. En esta estimación también se han introducido algunas de las variables previas de profesionalización que en estudios sobre determinantes de la productividad han sido consideradas por la literatura. Así, se han incluido las variables de inversión en investigación de mercado, formación de la mano de obra, uso de las TIC y si el principal cliente de la empresa es extranjero (como proxy de su actividad exportadora). De ellas, únicamente el uso de las TIC (para cualquier medida de productividad) y si el principal cliente es extranjero (para las dos medidas sencillas que no implican la estimación de la PTF) presentan signo positivo y significatividad estadística. Estas variables tienen, por tanto, una relación directa con la productividad más allá de su potencial efecto previo sobre las decisiones de I+D y de gasto.

En el análisis de la última etapa de estimación en este trabajo se observa en general que, a medida que se tienden a utilizar indicadores de productividad del tipo PTF, el impacto de las variables explicativas disminuye.

Tras la realización de este estudio, se pone de manifiesto la importancia de fomentar por parte del sector público el uso de prácticas empresariales ligadas a una mayor profesionalización/modernización de las empresas e internacionalización de 
las mismas, lo que contribuye de forma directa y/o indirecta (a través de la inversión en I+D) a las mejoras de productividad de las empresas ecuatorianas. Para países en vías de desarrollo como es el caso de Ecuador, donde la intensidad en I+D es baja, resultan fundamentales este tipo de trabajos, pues sus resultados no solo son informativos para el gobierno sino también para las empresas, esperándose que de ellos se derive una mayor sensibilización de la economía en general hacia la innovación y la productividad.

\section{Referencias bibliográficas}

[1] AGHION, P. y HOWITT, P. (1992). «A model of growth through creative destruction». Econometrica, 60, 323-351.

[2] AGHION, P. y HOWITT, P. (1998). Endogenous Growth Theory. The MIT Press.

[3] AMBEC, S., COHEN, M., ELGIE, S. y LANOIE, P. (2013). «The Porter Hypothesis at 20: Can Environmental Regulation Enhance Innovation and Competitiveness?». Review of Environmental Economics and Policy, 7(1), 2-22.

[4] AÑÓN,D.,MANJÓN,M. y MAÑEZ,J. (2011). «Multinationals, R\&D, and productivity: evidence for UK manufacturing firms». Industrial and Corporate Change, 20(2), 641659.

[5] AÑÓN, D. y MANJÓN, M. (2009). «Does Internationalization alter the R\&D productivity relationship?». Documento de Trabajo, Universitat Rovira i Virgili. Departament d'Economia.

[6] BENAVENTE, J. M. (2006). «The role of research and innovation in promoting productivity in Chile». Economics of Innovation and New Technology, 55, 301-315.

[7] BRATTI, M. y FELICE, G. (2012). «Are exporters more likely to introduce product innovation?». The World Economy, 35, 1559-1598.

[8] CHUDNOVSKY, D., LÓPEZ, A. y PUPATO, G. (2006). «Innovation and productivity in developing countries: A study of Argentine manufacturing firms's behavior (19922001)». Research Policy, 35, 266-288.

[9] LEY DE DEFENSA DEL ARTESANO (1998). http://www.artesanos.gob.ec/wpcontent/uploads/downloads/2014/05/ley_defensa_artesano.pdf.

[10] CRÉPON, B., DUGUET, E. y MAIRESSE, J. (1998). «Research and Development, Innovation and Productivity: An Econometric Analysis at the Firm Level». Economics of Innovation and New Technology, 7(2), 115-158.

[11] CRESPI, G. y ZÚÑIGA, P. (2012). «Innovation and productivity: evidence from six Latin American countries». World Development, 40(2), 273-290.

[12] DORASZELSKI, U. y JAUMANDREU, J. (2013). «R\&D and Productivity: Estimating endogenous productivity». Review of Economic Studies, 80(4), 1338-1283.

[13] GRILICHES, Z. (1970). «R\&D and the productivity slowdown». American Economic Review, 2, 343-348.

[14] GRILICHES, Z. (1979). «Issues in assessing the contribution of R\&D to productivity growth». Bell Journal of Economics, 10, 92-116.

[15] HALL, B. (2011). «Innovation and productivity». UNIT-MERIT Working Paper Series 2011-028. 
[16] HALL, B., LOTTI, F. y MAIRESSE, J. (2009). «Innovation and productivity in SMEs: empirical evidence for Italy». Small Business Economics, 33, 13-33.

[17] HECKMAN, J. (1979). «Sample Selection Bias as a Specification Error». Econometrica, 47(1), 153-161.

[18] LEVINSOHN, J. y PETRIN, A. (2003). «Estimating production functions using inputs to control for unobservables». Review of Economic Studies, 70, 317-342.

[19] MÁÑEZ, J.A., ROCHINA-BARRACHINA, M.E. y Sanchis-Llopis, J.A. (2006). «The decision to invest in R\&D: a panel data analysis for Spanish manufacturing». International Journal of Applied Economics, 3, 80-94.

[20] MÁÑEZ, J.A., ROCHINA-BARRACHINA, M.E. y SANCHIS-LLOPIS, J.A. (2009). «Self-selection into exports: Productivity and/or innovation?». Applied Economics Quarterly, 55, 219-242.

[21] MÁÑEZ, J.A., ROCHINA-BARRACHINA, M.E. y SANCHIS-LLOPIS, J.A. (2015). «The Dynamic linkages among exports, R\&D and Productivity». World Economy, 38, 583-612.

[22] OLLEY, G. S. y PAKES, A. (1996). «The dynamics of productivity in the telecommunications equipment industry». Econometrica, 64, 1263-1297.

[23] OLLO-LÓPEZ, A. y ARAMENDÍA-MUNETA, M.E. (2012). «ICT impact on competitiveness, innovation and environment». Telematics and Informatics, 29, 204-210.

[24] PARISI, M.L., SCHIANTARELLI, F. y Sembenelli,A. (2006). «Productivity, innovation and R\&D Microevidence for Italy». European Economic Review, 50, 2037-2061.

[25] PORTER, M. y VAN DER LINDE, C. (1995). «Toward a New conception of the enviromental-competitiveness relationship». Journal of Economic Perspectives, 9(4), 97-118.

[26] SCHUMPETER, J. (1942). Capitalism, Socialism and Democracy. New York: Harper y Bros.

[27] SOLOW, R. M. (1957). «Technical change and teh aggregate production function». The Review of Economics and Statistics, 39, 312-320.

[28] THORNHILL, S. (2006). «Knowledge, innovation and firm perfomance in high and low technology regimes». Journal of Business Venturing, 21, 687-703.

[29] TOBIN, J. (1958). «Estimation of Relationships for Limited Dependent Variables». Econometrica, 26, 24-36. 


\section{ANEXOS}

ANEXO 1. SECTORES

\begin{tabular}{|l|l|}
\hline Sector & \multicolumn{1}{|c|}{ Descripción } \\
\hline Sector 1 & Alimentación, Bebidas y Tabaco \\
\hline Sector 2 & Textiles \\
\hline Sector 3 & Cuero y Calzado \\
\hline Sector 4 & Madera y Papel \\
\hline Sector 5 & Productos Químicos y Derivados del Petróleo \\
\hline Sector 6 & Plástico y Caucho \\
\hline Sector 7 & Cristal y Productos no Metálicos \\
\hline Sector 8 & Muebles \\
\hline Sector 9 & Productos Metálicos \\
\hline Sector 10 & Maquinaria de Oficina, Eléctrica, y Otra Maquinaria \\
\hline Sector 11 & Aparatos de Comunicación, Médicos, Precisión y Ópticos \\
\hline Sector 12 & Equipos de Transporte \\
\hline
\end{tabular}

ANEXO 2. DEFINICIÓN DE LAS VARIABLES EXPLICATIVAS

\begin{tabular}{|l|l|}
\hline \multicolumn{1}{|c|}{ Variable } & \multicolumn{1}{|c|}{ Descripción } \\
\hline Provincia & $\begin{array}{l}\text { Dummy para cada provincia (24 provincias) y una variable } \\
\text { identificada como zona no delimitada para territorios en } \\
\text { conflicto hasta el momento de la encuesta. }\end{array}$ \\
\hline Sectores & $\begin{array}{l}\text { Cada uno de los sectores se encuentran en el Anexo 1 y fueron } \\
\text { transformados a dicotómicas donde 1 representa pertenecer } \\
\text { al sector y 0 en otro caso. }\end{array}$ \\
\hline Personas naturales & $\begin{array}{l}\text { Dummy que toma valores de 1 si la empresa pertenece a una } \\
\text { persona natural y 0 en otro caso. }\end{array}$ \\
\hline Empresa sin fines de lucro & $\begin{array}{l}\text { Dummy que toma valores de 1 si la empresa no tiene fines de } \\
\text { lucro y 0 en otro caso. }\end{array}$ \\
\hline Empresa privada & $\begin{array}{l}\text { Dummy que toma valores de 1 si la empresa es privada y 0 } \\
\text { en otro caso. }\end{array}$ \\
\hline Empresa de control extranjero & $\begin{array}{l}\text { Dummy que toma el valor de 1 si la empresa es de control } \\
\text { extranjero y 0 en otro caso. }\end{array}$ \\
\hline Empresa pública & $\begin{array}{l}\text { Se denomina Empresa Pública a la empresa que tiene control } \\
\text { del Gobierno Central (Petróleos y sus derivados son las } \\
\text { principales en el país). Dummy que toma valores de 1 si la } \\
\text { empresa es Pública y 0 en otro caso. }\end{array}$ \\
\hline Empresa de gobierno local & $\begin{array}{l}\text { Se denomina Empresa de Gobierno local a la empresa que es } \\
\text { controlada por los gobiernos locales (prefecturas, municipios, } \\
\text { gobiernos autónomos) que recibe fondos del Presupuesto } \\
\text { general del Estado. Dummy que toma valores de 1 si la } \\
\text { empresa es del Gobierno local y 0 en otro caso. }\end{array}$ \\
\hline
\end{tabular}




\section{ANEXO 2. DEFINICIÓN DE LAS VARIABLES EXPLICATIVAS (continuación)}

\begin{tabular}{|c|c|}
\hline Variable & Descripción \\
\hline Cooperativa de Ahorro y Crédito & $\begin{array}{l}\text { La principal actividad de las empresas de «Ahorro y Crédito» } \\
\text { es entregar crédito a las personas y pequeñas empresas, pero } \\
\text { en muchos casos pueden realizar otras actividades (incluida la } \\
\text { manufacturera). Dummy que toma valores de } 1 \text { si la Empresa es } \\
\text { de Ahorro y Crédito y } 0 \text { en otro caso. }\end{array}$ \\
\hline Cooperativa & $\begin{array}{l}\text { Empresas registradas para realizar actividades específicas como } \\
\text { grupos cooperativos (Agricultura, Ganadería, entre otras, y sus } \\
\text { derivados). Dummy que toma valores de } 1 \text { si la Empresa es } \\
\text { Cooperativa y } 0 \text { en otro caso. }\end{array}$ \\
\hline Asociación & $\begin{array}{l}\text { Empresas NO registradas para realizar actividades específicas } \\
\text { como grupos cooperativos (Agricultura, Ganadería, entre otras, } \\
\text { y sus derivados). Dummy que toma valores de } 1 \text { si la Empresa es } \\
\text { Asociación y } 0 \text { en otro caso. }\end{array}$ \\
\hline Red Empresarial & $\begin{array}{l}\text { Dummy que toma valores de } 1 \text { si la empresa pertenece a una red } \\
\text { empresarial y } 0 \text { en otro caso. }\end{array}$ \\
\hline Investigación de Mercado & $\begin{array}{l}\text { Dummy que toma valores de } 1 \text { si la empresa realizó actividades } \\
\text { de investigación de mercado y } 0 \text { en otro caso. }\end{array}$ \\
\hline Contabilidad & $\begin{array}{l}\text { Dummy que toma valores de } 1 \text { si la empresa lleva contabilidad y } \\
0 \text { en otro caso. }\end{array}$ \\
\hline Financiamiento & $\begin{array}{l}\text { Dummy que toma valores de } 1 \text { si la empresa obtuvo financiamiento } \\
\text { externo y } 0 \text { en otro caso. }\end{array}$ \\
\hline Medio ambiente & $\begin{array}{l}\text { Dummy que toma valores de } 1 \text { si la empresa realizó actividades } \\
\text { de mejora del medio ambiente y } 0 \text { en otro caso. }\end{array}$ \\
\hline Formación & $\begin{array}{l}\text { Dummy que toma valores de } 1 \text { si la empresa realiza actividades de } \\
\text { formación o capacitación para los empleados y } 0 \text { en otro caso. }\end{array}$ \\
\hline TIC & $\begin{array}{l}\text { Dummy que toma valores de } 1 \text { si la empresa utiliza Tecnologías } \\
\text { de la Información y Comunicación y } 0 \text { en otro caso. }\end{array}$ \\
\hline Principal cliente extranjero & $\begin{array}{l}\text { Dummy que toma valores de } 1 \text { si el principal cliente de la empresa } \\
\text { es extranjero y } 0 \text { en otro caso. }\end{array}$ \\
\hline Calificación Artesanal & $\begin{array}{l}\text { Si la empresa tiene calificación artesanal. Este certificado es } \\
\text { entregado por la Junta Nacional del Artesano después de cumplir } \\
\text { ciertos criterios que establece la Ley de Defensa al Artesano. Las } \\
\text { personas naturales o jurídicas que obtengan esta certificación } \\
\text { tienen beneficios tributarios. Dummy que toma valores } 1 \text { si la } \\
\text { empresa tiene la certificación y } 0 \text { en otro caso. }\end{array}$ \\
\hline Local Propio & $\begin{array}{l}\text { Dummy que toma valores de } 1 \text { si la empresa cuenta con local } \\
\text { propio y } 0 \text { en otro caso. }\end{array}$ \\
\hline Empresa Matriz & $\begin{array}{l}\text { Dummy que toma valores de } 1 \text { si la empresa es la matriz y } 0 \text { en } \\
\text { otro caso. }\end{array}$ \\
\hline Gerente hombre & $\begin{array}{l}\text { Dummy que toma valores de } 1 \text { si el gerente de la empresa es } \\
\text { hombre y } 0 \text { en otro caso. }\end{array}$ \\
\hline Log trabajadores & El número de trabajadores de la empresa en logaritmos. \\
\hline$(\text { Log trabajadores })^{2}$ & $\begin{array}{l}\text { El cuadrado del logaritmo del número de trabajadores de la } \\
\text { empresa. }\end{array}$ \\
\hline Log edad & Número de años desde que la empresa fue fundada en logaritmos. \\
\hline Log capital por trabajador & Ratio de capital por trabajador en logaritmos. \\
\hline
\end{tabular}


ANEXO 3. DESCRIPTIVOS DE LAS VARIABLES IMPLICADAS EN EL ANÁLISIS

\begin{tabular}{|c|c|c|c|c|c|}
\hline Variable & Obs. & Media & Desv. típica & Mínimo & Máximo \\
\hline $\mathrm{Si} / \mathrm{No} \mathrm{I}+\mathrm{D}$ & 42292 & 0,0099113 & 0,0990621 & 0 & 1 \\
\hline Log gasto I+D & 42292 & 0,0765477 & 0,8021128 & 0 & 17,47994 \\
\hline Azuay & 42292 & 0,10112 & 0,3014913 & 0 & 1 \\
\hline Bolívar & 42292 & 0,0077804 & 0,0878637 & 0 & 1 \\
\hline Cañar & 42292 & 0,0196987 & 0,1389645 & 0 & 1 \\
\hline Carchi & 42292 & 0,0078051 & 0,0880024 & 0 & 1 \\
\hline Cotopaxi & 42292 & 0,0294613 & 0,1690978 & 0 & 1 \\
\hline Chimborazo & 42292 & 0,0392239 & 0,1941298 & 0 & 1 \\
\hline El Oro & 42292 & 0,0376381 & 0,1903219 & 0 & 1 \\
\hline Esmeraldas & 42292 & 0,0165023 & 0,1273985 & 0 & 1 \\
\hline Guayas & 42292 & 0,1918331 & 0,3937474 & 0 & 1 \\
\hline Imbabura & 42292 & 0,0383319 & 0,1919987 & 0 & 1 \\
\hline Loja & 42292 & 0,0385549 & 0,1925341 & 0 & 1 \\
\hline Los Ríos & 42292 & 0,0296843 & 0,1697171 & 0 & 1 \\
\hline Manabí & 42292 & 0,0558006 & 0,2295391 & 0 & 1 \\
\hline Morona Santiago & 42292 & 0,0096883 & 0,0979524 & 0 & 1 \\
\hline Napo & 42292 & 0,0045592 & 0,0673685 & 0 & 1 \\
\hline Pastaza & 42292 & 0,0064919 & 0,0803113 & 0 & 1 \\
\hline Pichincha & 42292 & 0,2394569 & 0,4267573 & 0 & 1 \\
\hline Tungurahua & 42292 & 0,060112 & 0,2376972 & 0 & 1 \\
\hline Zamora & 42292 & 0,0069627 & 0,0831527 & 0 & 1 \\
\hline Galápagos & 42292 & 0,0019823 & 0,044479 & 0 & 1 \\
\hline Sucumbíos & 42292 & 0,0078795 & 0,0884171 & 0 & 1 \\
\hline Orellana & 42292 & 0,0050548 & 0,0709178 & 0 & 1 \\
\hline Santo Domingo & 42292 & 0,0296843 & 0,1697171 & 0 & 1 \\
\hline Santa Elena & 42292 & 0,0141979 & 0,1183076 & 0 & 1 \\
\hline Zonas No delimitadas & 42292 & 0,0004956 & 0,022256 & 0 & 1 \\
\hline Alimentación & 42292 & 0,2214679 & 0,4152398 & 0 & 1 \\
\hline Textiles & 42292 & 0,2188166 & 0,4134491 & 0 & 1 \\
\hline Calzado y Piel & 42292 & 0,0276773 & 0,1640485 & 0 & 1 \\
\hline Madera y papel & 42292 & 0,0991377 & 0,2988505 & 0 & 1 \\
\hline Químico & 42292 & 0,0090193 & 0,0945418 & 0 & 1 \\
\hline Caucho y Plástico & 42292 & 0,0110511 & 0,104543 & 0 & 1 \\
\hline Prod. no metálicos & 42292 & 0,0578572 & 0,2334761 & 0 & 1 \\
\hline
\end{tabular}




\section{ANEXO 3. DESCRIPTIVOS DE LAS VARIABLES IMPLICADAS EN EL ANÁLISIS (continuación)}

\begin{tabular}{|c|c|c|c|c|c|}
\hline Variable & Obs. & Media & Desv. típica & Mínimo & Máximo \\
\hline Muebles & 42292 & 0,1603895 & 0,3669715 & 0 & 1 \\
\hline Prod. metálicos & 42292 & 0,1662372 & 0,372298 & 0 & 1 \\
\hline Maq. ofic. y elect. & 42292 & 0,0131077 & 0,1137374 & 0 & 1 \\
\hline Com./prec./méd & 42292 & 0,0052034 & 0,0719478 & 0 & 1 \\
\hline Eq. de transporte & 42292 & 0,0100352 & 0,0996731 & 0 & 1 \\
\hline Empresa sin fines de Lucro & 42292 & 0,000669 & 0,0258569 & 0 & 1 \\
\hline Empresa privada & 42292 & 0,0372665 & 0,1894164 & 0 & 1 \\
\hline Empresa de control ext. & 42292 & 0,0001487 & 0,0121923 & 0 & 1 \\
\hline Empresa pública & 42292 & 0,0003965 & 0,0199074 & 0 & 1 \\
\hline Empresa de gobierno local & 42292 & 0,0001239 & 0,0111301 & 0 & 1 \\
\hline Cooperativa de Aho. y Créd. & 42292 & 0,0000248 & 0,0049778 & 0 & 1 \\
\hline Cooperativa & 42292 & 0,0002478 & 0,0157393 & 0 & 1 \\
\hline Asociación & 42292 & 0,0018336 & 0,0427817 & 0 & 1 \\
\hline Personas Naturales & 42292 & 0,9592894 & 1,1976215 & 0 & 1 \\
\hline Red empresarial & 42292 & 0,1931959 & 0,3948102 & 0 & 1 \\
\hline Inv. de Mercado & 42292 & 0,0240101 & 0,1530823 & 0 & 1 \\
\hline Contabilidad & 42292 & 0,0792408 & 0,2701176 & 0 & 1 \\
\hline Financiamiento & 42292 & 0,2468903 & 0,4312077 & 0 & 1 \\
\hline Medio ambiente & 42292 & 0,0175182 & 0,1311936 & 0 & 1 \\
\hline Formación & 42292 & 0,0436097 & 0,2042276 & 0 & 1 \\
\hline TIC & 42292 & 0,099782 & 0,2997128 & 0 & 1 \\
\hline Principal cliente ext. & 42292 & 0,0056247 & 0,0747874 & 0 & 1 \\
\hline Calificación artesanal & 42292 & 0,2960008 & 0,456497 & 0 & 1 \\
\hline Local propio & 42292 & 0,4731899 & 0,4992869 & 0 & 1 \\
\hline Empresa Matriz & 42292 & 0,0342187 & 0,1817928 & 0 & 1 \\
\hline Gerente hombre & 42292 & 0,7442143 & 0,4363073 & 0 & 1 \\
\hline Log trabajadores & 42292 & 0,7539823 & 0,8430514 & 0 & 8,540519 \\
\hline$(\text { Log trabajadores })^{2}$ & 42292 & 1,279207 & 3,254389 & 0 & 72,94046 \\
\hline Log edad & 42292 & 1,732824 & 1,084746 & 0 & 4,94876 \\
\hline Log capital por trab. & 40358 & 7,363451 & 1,403244 & $-1,098612$ & 17,32963 \\
\hline Log Produc. por trabajador & 40358 & 8,89816 & 1,028596 & 3,688879 & 19,31963 \\
\hline Log VA por trabajador & 40358 & 8,268483 & 1,12628 & $-9,574634$ & 19.31523 \\
\hline Log PTF & 40358 & 0,0459322 & 0,5869696 & -2.359767 & 7,157747 \\
\hline Log PTF_VA & 40358 & $-0,0120145$ & 1,011565 & -18.02968 & 9,032396 \\
\hline
\end{tabular}

